\title{
PROOF-RELEVANT LOGICAL RELATIONS FOR NAME GENERATION
}

\author{
NICK BENTON, MARTIN HOFMANN, AND VIVEK NIGAM
}

Facebook, London, UK

e-mail address: nick.benton@gmail.com

LMU, Munich, Germany

e-mail address: hofmann@ifi.lmu.de

UFPB, João Pessoa, Brazil \& fortiss, Munich, Germany

e-mail address: vivek.nigam@gmail.com

\begin{abstract}
Pitts and Stark's $v$-calculus is a paradigmatic total language for studying the problem of contextual equivalence in higher-order languages with name generation. Models for the $v$-calculus that validate basic equivalences concerning names may be constructed using functor categories or nominal sets, with a dynamic allocation monad used to model computations that may allocate fresh names. If recursion is added to the language and one attempts to adapt the models from (nominal) sets to (nominal) domains, however, the direct-style construction of the allocation monad no longer works. This issue has previously been addressed by using a monad that combines dynamic allocation with continuations, at some cost to abstraction.

This paper presents a direct-style model of a $v$-calculus-like language with recursion using the novel framework of proof-relevant logical relations, in which logical relations also contain objects (or proofs) demonstrating the equivalence of (the semantic counterparts of) programs. Apart from providing a fresh solution to an old problem, this work provides an accessible setting in which to introduce the use of proof-relevant logical relations, free of the additional complexities associated with their use for more sophisticated languages.
\end{abstract}

\section{INTRODUCTION}

Reasoning about contextual equivalence in higher-order languages that feature dynamic allocation of names, references, objects or keys is challenging. Pitts and Stark's $v$-calculus boils the problem down to its purest form, being a total, simply-typed lambda calculus with just names and booleans as base types, an operation new that generates fresh names, and equality testing on names. The full equational theory of the $v$-calculus is surprisingly complex and has been studied both operationally and denotationally, using logical relations [Sta94, PS98], environmental bisimulations [BK13] and nominal game semantics $\left[\mathrm{AGM}^{+} 04\right.$, Tze12].

Key words and phrases: logical relations, parametricity, program transformation.

A preliminary version of this work was presented at the 11th International Conference on Typed Lambda Calculi and Applications, TLCA 2013, 26-28 June 2013, Eindhoven, The Netherlands.

LOGICAL METHODS 5. IN COMPUTER SCIENCE
DOI:10.23638/LMCS-14(1:25)2018
(C) Nick Benton et al.

(c) Creative Commons 
Even before one considers the 'exotic' equivalences that arise from the (partial) encapsulation of names within closures, there are two basic equivalences that hold for essentially all forms of generativity:

$$
\begin{array}{ll}
(\text { let } x \Leftarrow \text { new in } e)=e, \text { provided } x \text { is not free in } e . & \text { (Drop) } \\
(\text { let } x \Leftarrow \text { new in let } y \Leftarrow \text { new in } e)=(\text { let } y \Leftarrow \text { new in let } x \Leftarrow \text { new in } e) \quad \text { (Swap). }
\end{array}
$$

The (Drop) equivalence says that removing the generation of unused names preserves behaviour; this is sometimes called the 'garbage collection' rule. The (Swap) equivalence says that the order in which names are generated is immaterial. These two equations also appear as structural congruences for name restriction in the $\pi$-calculus.

Denotational models for the $v$-calculus validating (Drop) and (Swap) may be constructed using (pullback-preserving) functors in $\mathrm{Set}^{\mathbf{W}}$, where $\mathbf{W}$ is the category of finite sets and injections [Sta94], or in FM-sets [GP02]. These models use a dynamic allocation monad to interpret possibly-allocating computations. One might expect that moving to $\mathrm{Cpo}^{\mathbf{W}}$ or FM-cpos would allow such models to adapt straightforwardly to a language with recursion, and indeed Shinwell, Pitts and Gabbay originally proposed [SPG03] a dynamic allocation monad over FM-cpos. However, it turned out that the underlying FM-cppo of the proposed monad does not actually have least upper bounds for all finitelysupported chains. A counter-example is given in Shinwell's thesis [Shi04, page 86]. To avoid the problem, Shinwell and Pitts [Shi04, SP05] moved to an indirect-style model, using a continuation monad [PS98]: $(-)^{\mathrm{TT}} \stackrel{\text { def }}{=}\left(-\rightarrow 1_{\perp}\right) \rightarrow 1_{\perp}$ to interpret computations. In particular, one shows that two programs are equivalent by proving that they co-terminate when supplied with the same (or equivalent) continuations. The CPS approach was also adopted by Benton and Leperchey [BL05], and by Bohr and Birkedal [BB06], for modelling languages with references.

In the context of our on-going research on the semantics of effect-based program transformations [BKHB06], we have been led to develop proof-relevant logical relations [BHN14]. These interpret types not merely as partial equivalence relations, as is commonly done, but as a proof-relevant generalization thereof: setoids. A setoid is like a category all of whose morphisms are isomorphisms (a groupoid) with the difference that no equations between these morphisms are imposed. The objects of a setoid establish that values inhabit semantic types, whilst its morphisms are understood as explicit proofs of semantic equivalence. This paper shows how we can use proof-relevant logical relations to give a direct-style model of a language with name generation and recursion, validating (Drop) and (Swap). Apart from providing a fresh approach to an old problem, our aim in doing this is to provide a comparatively accessible presentation of proof-relevant logical relations in a simple setting, free of the extra complexities associated with specialising them to abstract regions and effects [BHN14].

Although our model validates the two most basic equations for name generation, it is - like simple functor categories in the total case - still far from fully abstract. Many of the subtler contextual equivalences of the $v$-calculus still hold in the presence of recursion; one naturally wonders whether the more sophisticated methods used to prove those equivalences carry over to the proof-relevant setting. We will show one such method, Stark's parametric functors, which are a categorical version of Kripke logical relations, does indeed generalize smoothly, and can be used to establish a non-trivial equivalence involving encapsulation of fresh names. Moreover, the proof-relevant version is naturally transitive, which is, somewhat notoriously, not generally true of ordinary logical relations.

Section 1 sketches the language with which we will be working, and a naive 'raw' domaintheoretic semantics for it. This semantics does not validate interesting equivalences, but is adequate. By constructing a realizability relation between it and the more abstract semantics we subsequently introduce, we will be able to show adequacy of the more abstract semantics. In Section 2 we 


$$
\begin{aligned}
& \overline{\Gamma, x: \tau \vdash_{v} x: \tau} \quad \overline{\Gamma \vdash_{v} b: \text { bool }} \quad \overline{\Gamma \vdash_{v} i: \text { int }} \quad \frac{\Gamma, f: \tau \rightarrow \tau^{\prime}, x: \tau \vdash_{c} e: \tau^{\prime}}{\Gamma \vdash_{v} \operatorname{rec} f x=e: \tau \rightarrow \tau^{\prime}} \\
& \frac{\Gamma \vdash_{v} v: \text { int } \quad \Gamma \vdash_{v} v^{\prime}: \text { int }}{\Gamma \vdash_{v} v+v^{\prime}: \text { int }} \quad \frac{\Gamma \vdash_{v} v: \tau \quad \Gamma \vdash_{v} v^{\prime}: \tau \quad \tau \in\{\text { int, name }\}}{\Gamma \vdash_{v} v=v^{\prime}: \text { bool }} \quad \frac{\Gamma \vdash_{v} v: \tau}{\Gamma \vdash_{c} v: \tau} \\
& \overline{\Gamma \vdash_{c} \text { new : name }} \quad \frac{\Gamma \vdash_{c} e: \tau \quad \Gamma, x: \tau \vdash_{c} e^{\prime}: \tau^{\prime}}{\Gamma \vdash_{c} \text { let } x \Leftarrow e \text { in } e^{\prime}: \tau^{\prime}} \quad \frac{\Gamma \vdash_{v} v: \tau \rightarrow \tau^{\prime} \quad \Gamma \vdash_{v} v^{\prime}: \tau}{\Gamma \vdash_{c} v v^{\prime}: \tau^{\prime}} \\
& \frac{\Gamma \vdash_{v} v: \text { bool } \quad \Gamma \vdash_{c} e: \tau \quad \Gamma \vdash_{c} e^{\prime}: \tau}{\Gamma \vdash_{c} \text { if } v \text { then } e \text { else } e^{\prime}: \tau}
\end{aligned}
$$

Figure 1: Typing rules for language with recursion and name generation.

introduce our category of setoids; these are predomains where there is a (possibly-empty) set of 'proofs' witnessing the equality of each pair of elements. We then describe pullback-preserving functors from the category of worlds $\mathbf{W}$ into the category of setoids. Such functors will interpret types of our language in the more abstract semantics, with morphisms between them interpreting terms. The interesting construction here is that of a dynamic allocation monad over the category of pullback-preserving functors. Section 7 shows how the abstract semantics is defined and related to the more concrete one. Section 8 then shows how the semantics may be used to establish basic equivalences involving name generation. Section 9 describes how proof-relevant parametric functors can validate a more subtle equivalence involving encapsulation of new names.

\section{Syntax and Semantics}

We work with an entirely conventional CBV language, featuring recursive functions and base types that include names, equipped with equality testing and fresh name generation (here + is just a representative operation on integers):

$$
\begin{aligned}
\tau & :=\text { int } \mid \text { bool } \mid \text { name } \mid \tau \rightarrow \tau^{\prime} \\
v & :=x|b| i|\operatorname{rec} f x=e| v+v^{\prime} \mid v=v^{\prime} \\
e & :=v \mid \text { new } \mid \text { let } x \Leftarrow e \text { in } e^{\prime}\left|v v^{\prime}\right| \text { if } v \text { then } e \text { else } e^{\prime} \\
\Gamma & :=x_{1}: \tau_{1}, \ldots, x_{n}: \tau_{n}
\end{aligned}
$$

The expression rec $f x=e$ stands for an anonymous function which satisfies the recursive equation $f(x)=e$ where both $x$ and $f$ may occur in $e$. In the special case where $f$ does not occur in $e$, the construct degenerates to function abstraction. We thus introduce the abbreviation:

$$
\text { fun } x . e \triangleq \operatorname{rec} f x=e \quad \text { where } f \text { does not occur in } e \text {. }
$$

There are typing judgements for values, $\Gamma \vdash_{v} v: \tau$, and computations, $\Gamma \vdash_{c} e: \tau$, defined in an unsurprising way; these are shown in Figure 1. We will often elide the subscript on turnstiles. 
We define a simple-minded concrete denotational semantics $\llbracket \cdot \pi$ for this language using predomains ( $\omega$-cpos) and continuous maps. For types we take

$$
\begin{aligned}
& \| \text { int }\|=\mathbb{Z} \quad\| \text { bool } \|=\mathbb{B} \quad \text { nname } \|=\mathbb{N} \\
& \left\|\tau \rightarrow \tau^{\prime} \rrbracket=\llbracket \tau\right\| \rightarrow\left(\mathbb{N} \rightarrow \mathbb{N} \times\left\|\tau^{\prime}\right\|\right)_{\perp} \\
& \llbracket x_{1}: \tau_{1}, \ldots, x_{n}: \tau_{n} \rrbracket=\llbracket \tau_{1} \rrbracket \times \cdots \times \| \tau_{n} \rrbracket
\end{aligned}
$$

and there are then conventional clauses defining

$$
\| \Gamma \vdash_{v} v: \tau \rrbracket: \pi \Gamma \rrbracket \rightarrow \pi \tau \rrbracket \quad \text { and } \quad \llbracket \Gamma \vdash_{c} e: \tau \|: \llbracket \Gamma \rrbracket \rightarrow(\mathbb{N} \rightarrow \mathbb{N} \times \llbracket \tau \|)_{\perp}
$$

Note that this semantics just uses naturals to interpret names, and a state monad over names to interpret possibly-allocating computations. For allocation we take

$$
\llbracket \Gamma r_{c} \text { new : name } \|(\eta)=[\lambda n \cdot(n+1, n)]
$$

returning the next free name and incrementing the name supply. This semantics validates no interesting equivalences involving names, but is adequate for the obvious operational semantics. Our more abstract semantics, $\llbracket \cdot \rrbracket$, will be related to $\llbracket \cdot \pi$ in order to establish its adequacy.

\section{SETOIDS}

We define the category of setoids, Std, to be the exact completion of the category of predomains, see [CFS87, BCRS98]. We give here an elementary description of this category using the language of dependent types. A setoid $A$ consists of a predomain $|A|$ and for any two $x, y \in|A|$ a set $A(x, y)$ of "proofs" (that $x$ and $y$ are equal). The set of triples $X=\{(x, y, p) \mid p \in A(x, y)\}$ must itself be a predomain, i.e., there has to be an order relation $\leq \operatorname{such}$ that $(X, \leq)$ is a predomain. The first and second projections out of the set of triples must be continuous. Furthermore, there are continuous functions $r_{A}: \Pi x \in|A| \cdot A(x, x)$ and $s_{A}: \Pi x, y \in|A| \cdot A(x, y) \rightarrow A(y, x)$ and $t_{A}: \Pi x, y, z \cdot A(x, y) \times A(y, z) \rightarrow A(x, z)$, witnessing reflexivity, symmetry and transitivity; note that, unlike the case of groupoids, no equations involving $r, s$ and $t$ are imposed.

We should explain what continuity of a dependent function like $t(-,-)$ is: if $\left(x_{i}\right)_{i}$ and $\left(y_{i}\right)_{i}$ and $\left(z_{i}\right)_{i}$ are ascending chains in $A$ with suprema $x, y, z$ and $p_{i} \in A\left(x_{i}, y_{i}\right)$ and $q_{i} \in A\left(y_{i}, z_{i}\right)$ are proofs such that $\left(x_{i}, y_{i}, p_{i}\right)_{i}$ and $\left(y_{i}, z_{i}, q_{i}\right)_{i}$ are ascending chains, too, with suprema $(x, y, p)$ and $(y, z, q)$ then $\left(x_{i}, z_{i}, t\left(p_{i}, q_{i}\right)\right)$ is an ascending chain of proofs (by monotonicity of $\left.t(-,-)\right)$ and its supremum is $(x, z, t(p, q))$. Formally, such dependent functions can be reduced to non-dependent ones using pullbacks, that is $t$ would be a function defined on the pullback of the second and first projections from $\{(x, y, p) \mid p \in A(x, y)\}$ to $|A|$, but we find the dependent notation to be much more readable. If $p \in A(x, y)$ we may write $p: x \sim y$ or simply $x \sim y$. We also omit $\mid-1$ wherever appropriate. We remark that "setoids" also appear in constructive mathematics and formal proof, see e.g., [BCP03], but the proof-relevant nature of equality proofs is not exploited there and everything is based on sets (types) rather than predomains. A morphism from setoid $A$ to setoid $B$ is an equivalence class of pairs $f=\left(f_{0}, f_{1}\right)$ of continuous functions where $f_{0}:|A| \rightarrow|B|$ and $f_{1}: \Pi x, y \in|A| . A(x, y) \rightarrow B\left(f_{0}(x), f_{0}(y)\right)$. Two such pairs $f, g: A \rightarrow B$ are identified if there exists a continuous function $\mu: \Pi a \in|A| \cdot B\left(f_{0}(a), g_{0}(a)\right)$.

The following is folklore, see also [BCRS98].

Proposition 2.1. The category of setoids is cartesian closed. Cartesian product is given pointwise. The function space $A \Rightarrow B$ of setoids $A$ and $B$ is given as follows: the underlying predomain $|A \Rightarrow B|$ comprises pairs $\left(f_{0}, f_{1}\right)$ which are representatives of morphisms from $A$ to $B$. That is, $f_{0}:|A| \rightarrow|B|$ and $f_{1}: \Pi x, y \in|A| . A(x, y) \rightarrow B\left(f_{0}(x), f_{0}(y)\right)$ are continuous functions with the pointwise ordering. 
The proof set $(A \Rightarrow B)\left(\left(f_{0}, f_{1}\right),\left(f_{0}^{\prime}, f_{1}^{\prime}\right)\right)$ comprises witnesses of the equality of $\left(f_{0}, f_{1}\right)$ and $\left(f_{0}^{\prime}, f_{1}^{\prime}\right)$ qua morphisms, i.e., continuous functions $\mu: \Pi a \in|A| . B\left(f_{0}(a), f_{0}^{\prime}(a)\right)$.

Proof. The evaluation morphism $(A \Rightarrow B) \times A \longrightarrow B$ sends $\left(f_{0}, f_{1}\right)$ and $a$ to $f_{0}(a)$. If $h: C \times$ $A \longrightarrow B$ is a morphism represented by $\left(h_{0}, h_{1}\right)$ then the morphism $\lambda(h): C \longrightarrow A \Rightarrow B$ may be represented by $\left(\lambda(h)_{0}, \lambda(h)_{1}\right)$ where $\lambda(h)_{0}(c)=\left(f_{0}, f_{1}\right)$ and $f_{0}(a)=h_{0}(c, a)$ and $f_{1}\left(a, a^{\prime}, p\right)=$ $h_{1}\left((c, a),\left(c, a^{\prime}\right),(r(c), p)\right)$. Likewise, $\lambda(h)_{1}\left(c, c^{\prime}, p\right)=\mu$ where $\mu(a)=h_{1}\left((c, a),\left(c^{\prime}, a\right),(p, r(a))\right)$. The remaining verifications are left to the reader.

Definition 2.2. A setoid $D$ is pointed if $|D|$ has a least element $\perp$ and such that there is also a least proof $\perp \in D(\perp, \perp)$. If $D$ is pointed we write $\perp$ for the obvious global element $1 \rightarrow D$ returning $\perp$. A morphism $f: D \rightarrow D^{\prime}$ with $D, D^{\prime}$ both pointed is strict if $f \perp=\perp$.

Theorem 2.3. Let $D$ be a pointed setoid. Then there is a morphism of setoids $Y:[D \Rightarrow D] \rightarrow D$ satisfying the following equations (written using $\lambda$-calculus notation, which is meaningful in cartesian closed categories).

$$
\begin{array}{rlr}
f(Y(f)) & =Y(f) & \text { (Fixpoint) } \\
f(Y(g \circ f)) & =Y(f \circ g) & \text { (Dinaturality) } \\
f(Y(g)) & =Y(h) \text { if } f \text { is strict and } f g=h f & \text { (Uniformity) } \\
Y\left(f^{n}\right) & =Y(f) & \text { (Power) } \\
Y(\lambda x . f(x, x)) & =Y(\lambda x . Y(\lambda y . f(x, y))) & \text { (Diagonal) } \\
Y(\lambda \vec{x} \cdot \vec{t}(\vec{x})) & =\langle Y(s), \ldots, Y(s)\rangle & \text { (Amalgamation) } \\
\text { when } t_{i}(y, \ldots, y) & =s(y) \text { for } i=1, \ldots, n \text { and } \vec{t}=\left\langle t_{1}, \ldots, t_{n}\right\rangle &
\end{array}
$$

Proof. To define the morphism $Y$ suppose we are given $f=\left(f_{0}, f_{1}\right) \in|D \Rightarrow D|$. For each $i \in \mathbb{N}$ we define $d_{i} \in|D|$ by $d_{0}=\perp$ and $d_{i+1}=f_{0}\left(d_{i}\right)$. We then put $Y(f)=\sup _{i} d_{i}$.

Now suppose that $f^{\prime}=\left(f_{0}^{\prime}, f_{1}^{\prime}\right) \in|D \Rightarrow D|$ and $q: f \sim f^{\prime}$, i.e., $q: \Pi d . D\left(f_{0}(d), f_{0}^{\prime}(d)\right)$. Let $d_{i}^{\prime}$ be defined analogously to $d_{i}$ so that $Y\left(f^{\prime}\right)=\sup _{i} d_{i}^{\prime}$. By induction on $i$ we define proofs $p_{i}: d_{i} \sim d_{i}^{\prime}$. We put $p_{0}=\perp$ (the least proof) and, inductively, $p_{i+1}=t\left(f_{1}\left(p_{i}\right), q\left(d_{i}^{\prime}\right)\right)$ (transitivity). Notice that $f_{1}\left(p_{i}\right): d_{i+1} \sim f_{0}\left(d_{i}^{\prime}\right)$ and $q\left(d_{i}^{\prime}\right): f_{0}\left(d_{i}^{\prime}\right) \sim d_{i+1}^{\prime}$. Now let $\left(d, d^{\prime}, p\right)$ be the supremum of the chain $\left(d_{i}, d_{i}^{\prime}, p_{i}\right)$. By continuity of the projections we have that $d=Y(f)$ and $d^{\prime}=Y\left(f^{\prime}\right)$ and thus $p: Y(f) \sim Y\left(f^{\prime}\right)$. The passage from $q$ to $p$ witnesses that $Y$ is indeed a (representative of a) morphism.

Equations "Diagonal" and "Dinaturality" follow directly from the validity of these properties for the least fixpoint combinator for cpos. For the sake of completeness we prove the second one. Assume $f, g \in|D \Rightarrow D|$ and let $d_{i}=\left(f_{0} g_{0}\right)^{i}(\perp)$ and $e_{i}=\left(g_{0} f_{0}\right)^{i}(\perp)$. We have $d_{i} \leq f_{0}\left(e_{i}\right)$ and $\left.f_{0}\left(e_{i}\right)\right) \leq d_{i+1}$. It follows that $Y(f g)$ and $Y(g f)$ are actually equal. Equation "Fixpoint" is a direct consequence of dinaturality (take $g=\mathrm{id}$ ).

Amalgamation and uniformity are also valid for the least fixpoint combinator, but cannot be directly inherited since the equational premises only holds up to $\sim$. As a representative example we show amalgamation. So assume elements $t_{i} \in\left|D^{n} \Rightarrow D\right|$ and $s \in|D \Rightarrow D|$ and proofs $p_{k}$ : $\Pi d . D\left(\left(t_{k}\right)_{0}(d, \ldots, d), s(d)\right)$. Consider $d_{i}=\vec{t}_{0}^{t}(\perp, \ldots, \perp)$ and $e_{i}=s_{0}^{i}(\perp)$. By induction on $i$ and using the $p_{k}$ we construct proofs $d_{i} \sim\left(e_{i}, \ldots, e_{i}\right)$. The desired proof of $Y(\vec{t}) \sim(Y(s), \ldots, Y(s))$ is obtained as the supremum of these proofs as in the definition of the witness that $Y$ is a morphism above.

Equation "Power", finally, can be deduced from amalgamation and dinaturality or alternatively inherited directly from the least fixpoint combinator.

The above equational axioms for the fixpoint combinator are taken from Simpson and Plotkin [SP00], who show that they imply certain completeness properties. In particular, it follows that the 
category of setoids is an "iteration theory" in the sense of Bloom and Ésik [BÉ93]. For us they are important since the category of setoids is not cpo-enriched in any reasonable way, so that the usual order-theoretic characterisation of $Y$ is not available. Concretely, the equations help, for example, to justify various loop optimisations when loops are expressed using the fixpoint combinator.

Definition 2.4. A setoid $D$ is discrete if for all $x, y \in D$ we have $|D(x, y)| \leq 1$ and $|D(x, y)|=1 \Longleftrightarrow$ $x=y$.

Thus, in a discrete setoid proof-relevant equality and actual equality coincide and moreover any two equality proofs are actually equal (i.e. we have proof irrelevance).

\section{Finite SETS AND INJECTIONS}

Pullback squares are a central notion in our framework. As it will become clear later, they are the "proof-relevant" component of logical relations. Recall that a morphism $u$ in a category is a monomorphism if $u x=u x^{\prime}$ implies $x=x^{\prime}$ for all morphisms $x, x^{\prime}$. Two morphisms with common codomain are called a co-span and two morphisms with common domain are called span. A commuting square $x u=x^{\prime} u^{\prime}$ of morphisms is a pullback if whenever $x v=x^{\prime} v^{\prime}$ there is unique $t$ such that $v=u t$ and $v^{\prime}=u^{\prime} t$. This can be visualized as follows:

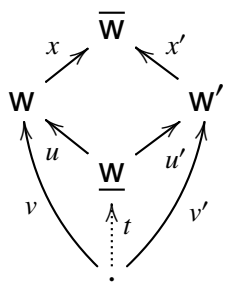

We write ${ }_{u}^{x} \diamond_{u^{\prime}}^{x^{\prime}}$ or $\mathbf{w}_{u}^{x} \diamond_{u^{\prime}}^{x^{\prime}} \mathbf{w}^{\prime}$ (when $\mathbf{w}^{(\prime)}=\operatorname{dom}\left(x^{(\prime)}\right)$ ) for such a pullback square. We call the common codomain of $x$ and $x^{\prime}$ the apex of the pullback, written $\overline{\mathrm{w}}$, while the common domain of $u, u^{\prime}$ is the low point of the square, written $\underline{\mathrm{w}}$. A pullback square $\mathrm{w}_{u}^{x} \diamond_{u^{\prime}}^{x^{\prime}} \mathrm{w}^{\prime}$ with apex $\overline{\mathrm{W}}$ is minimal if whenever there is another pullback $\mathbf{w}_{u}^{x_{1}} \diamond_{u^{\prime}}^{x_{1}^{\prime}} \mathbf{w}^{\prime}$ over the same span and with apex $\overline{\mathbf{w}_{1}}$, then there is a unique morphism $t: \overline{\mathrm{w}} \rightarrow \overline{\mathrm{w}_{1}}$ such that $x_{1}=t x$ and $x_{1}^{\prime}=t x^{\prime}$.

A category has pullbacks if every co-span can be completed to a pullback, which is necessarily unique up to isomorphism.

Definition 3.1. A category of worlds, $C$, is a category with pullbacks where any $\operatorname{span} u: \underline{\mathrm{w}} \rightarrow \mathrm{w}, u^{\prime}:$ $\underline{\mathrm{w}} \rightarrow \mathrm{w}^{\prime}$ can be completed to a minimal pullback square. Furthermore, there is a subcategory $\mathcal{I}$ of $C$ full on objects which is a poset, i.e., $|\mathcal{I}(X, Y)| \leq 1$. The morphisms in $\mathcal{I}$ are called inclusions. Moreover, any morphism $u$ in $C$ can be factored as $i_{1} ; u_{1}$ and as $u_{2} ; i_{2}$ where $i_{1}, i_{2}$ are inclusions and $u_{1}, u_{2}$ are isomorphisms.

Proposition 3.2. In a category of worlds all morphisms are monomorphisms and if $\mathbf{w}_{u}^{x} \diamond_{u^{\prime}}^{x^{\prime}} \mathbf{w}^{\prime}$ with ape $\overline{\mathrm{w}}$ is a minimal pullback then the morphisms $x$ and $x^{\prime}$ are jointly epic, i.e. for any $f, g: \overline{\mathrm{w}} \rightarrow \mathrm{w}_{1}$, if $f x=g x$ and $f x^{\prime}=g x^{\prime}$, then $f=g$.

Proof. First we show that any morphism $u: \mathrm{w} \rightarrow \mathrm{w}^{\prime}$ is a monomorphism. Let $\mathrm{w}^{\prime}{ }_{u}^{x} \diamond_{u}^{x^{\prime}} \mathrm{w}^{\prime}$ be a completion of the span $u, u$ to a (minimal) pullback. If $u a=u b=: h$, then $x h=x^{\prime} h$. So, the pullback property furnishes a unique map $c$ such that $u c=h$. Thus $c=a=b$, so $u$ is a monomorphism. 
Now suppose that $\mathbf{w}_{u}^{x} \diamond_{u^{\prime}}^{x^{\prime}} \mathbf{w}^{\prime}$ is a minimal pullback and $f x=g x=: h$ and $f x^{\prime}=g x^{\prime}=: h^{\prime}$. Then we claim that $\mathrm{w}^{\prime}{ }_{u} \diamond h_{u^{\prime}}^{h^{\prime}} \mathrm{w}^{\prime}$ is a pullback: if $h t=h^{\prime} t^{\prime}$, then since $f, g$ are monomorphisms by the above, we have $x t=x^{\prime} t^{\prime}$, so we can appeal to the pullback property of the original square.

Minimality of $\mathbf{w}^{\prime}{ }_{u} \diamond_{u}^{x^{\prime}} \mathbf{w}^{\prime}$ furnishes a unique map $k$ such that $h=k x$ and $h^{\prime}=k x^{\prime}$. But since $f$ and $g$ also have that property $\left(h=f x\right.$ and $h^{\prime}=f x^{\prime}$ and similarly for $g$ ), we conclude $f=g=k$.

Proposition 3.3. The category $\mathbf{W}$ with finite sets of natural numbers as objects and injective functions for morphisms and inclusions for the subcategory of inclusion $(\mathcal{I})$ is a category of worlds.

Proof. Given $f: X \rightarrow Z$ and $g: Y \rightarrow Z$ forming a co-span in $\mathbf{W}$, we form their pullback as $X \stackrel{f^{-1}}{\longleftarrow} f X \cap g Y \stackrel{g^{-1}}{\longrightarrow} Y$. This is minimal when $f X \cup g Y=Z$. Conversely, given a span $Y \stackrel{f}{\leftarrow} X \stackrel{g}{\rightarrow} Z$, we can complete to a minimal pullback by

$$
(Y \backslash f X) \uplus f X \stackrel{\left[i n_{1}, i n_{3} \circ f^{-1}\right]}{\longrightarrow}(Y \backslash f X)+(Z \backslash g X)+X \stackrel{\left[i n_{2}, i n_{3} \circ g^{-1}\right]}{\longleftarrow}(Z \backslash g X) \uplus g X
$$

where $[-,-]$ is case analysis on the disjoint union $Y=(Y \backslash f X) \uplus f X$. Thus a minimal pullback square in $\mathbf{W}$ is of the form:

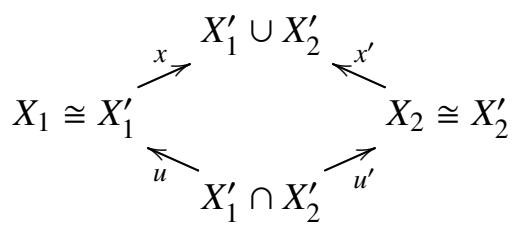

The factorization property is straightforward.

An object $\mathbf{w}$ of $\mathbf{W}$ models a set of generated/allocated names, with injective maps corresponding to renamings and extensions with newly generated names.

In $\mathbf{W}$, a minimal pullback corresponds to a partial bijection between $X_{1}$ and $X_{2}$, as used in other work on logical relations for generativity [PS93, BKBH07]. We write $u: x \hookrightarrow y$ to mean that $u$ is a subset inclusion and also use the notation $x \hookrightarrow y$ to denote the subset inclusion map from $x$ to $y$. Of course, the use of this notation implies that $x \subseteq y$. Note that if we have a span $u, u^{\prime}$ then we can choose $x, x^{\prime}$ so that ${ }_{u}^{x} \diamond_{u^{\prime}}^{x^{\prime}}$ is a minimal pullback and one of $x$ and $x^{\prime}$ is an inclusion. To do that, we simply replace the apex of any minimal pullback completion with an isomorphic one. The analogous property holds for completion of co-spans to pullbacks.

In this paper, we fix the category of worlds to be $\mathbf{W}$. The general definitions, in particular that of setoid-valued functors that we are going to give, also make sense in other settings. For example, in our treatment of proof-relevant logical relations for reasoning about stateful computation [BHN14], we build a category of worlds from partial equivalence relations on heaps.

\section{SETOID-VAluEd FUnCTORS}

A functor $A$ from the category of worlds $\mathbf{W}$ to the category of setoids comprises, as usual, for each $\mathrm{w} \in \mathbf{W}$ a setoid $A \mathrm{w}$, and for each $u: \mathrm{w} \rightarrow \mathrm{w}^{\prime}$ a morphism of setoids $A u: A \mathrm{w} \rightarrow A \mathrm{w}^{\prime}$ preserving identities and composition. This means that there exist continuous functions of type $\Pi a \cdot A \mathrm{w}(a,(A i d) a)$; and for any two morphisms $u: \mathrm{w} \rightarrow \mathrm{w}_{1}$ and $v: \mathrm{w}_{1} \rightarrow \mathrm{w}_{2}$ a continuous function of type $\Pi a \cdot A \mathrm{w}_{2}(A v(A u a), A(v u) a)$.

If $u: \mathrm{w} \rightarrow \mathrm{w}^{\prime}$ and $a \in A \mathrm{w}$ we may write $u . a$ or even $u a$ for $A u(a)$ and likewise for proofs in $A \mathrm{w}$. Note that there is a proof of equality of $(u v) . a$ and $u$.(v.a). In the sequel, we shall abbreviate 'setoid-valued functor(s)' as 'SVF(s)'. 
Intuitively, SVFs will become the denotations of types. Thus, an element of $A \mathrm{w}$ is a value involving at most the names in $\mathrm{w}$. If $u: \mathrm{w} \rightarrow \mathrm{w}_{1}$ then $A \mathrm{w} \ni a \mapsto u . a \in A \mathrm{w}_{1}$ represents renaming and possible weakening by names not "actually" occurring in $a$. Note that due to the restriction to injective functions identification of names ("contraction") is precluded. This is in line with Stark's use [Sta94] of set-valued functors on the category $\mathbf{W}$ to model fresh names.

Definition 4.1. We call an SVF, A, pullback-preserving if for every pullback square $\mathbf{w}_{u}^{x} \diamond_{u^{\prime}}^{x^{\prime}} \mathbf{w}^{\prime}$ with apex $\overline{\mathrm{w}}$ and low point $\underline{\mathrm{w}}$ the diagram $A \mathrm{w}_{A u}^{A x} \diamond_{A u^{\prime}}^{A x^{\prime}} A \mathrm{w}^{\prime}$ is a pullback in Std. This means that there is a continuous function of type

$$
\Pi a \in A \mathrm{w} . \Pi a^{\prime} \in A \mathrm{w}^{\prime} . A \overline{\mathrm{w}}\left(x . a, x^{\prime} . a^{\prime}\right) \rightarrow \Sigma \underline{a} \in A \underline{\mathrm{w}} \cdot A \mathrm{w}(u \cdot \underline{a}, a) \times A \mathrm{w}^{\prime}\left(u^{\prime} . \underline{a}, a^{\prime}\right)
$$

Thus, if two values $a \in A \mathrm{w}$ and $a^{\prime} \in A \mathrm{w}^{\prime}$ are equal in a common world $\overline{\mathrm{w}}$ then this can only be the case because there is a value in the "intersection world" $\underline{\mathrm{w}}$ from which both $a, a^{\prime}$ arise.

Note that the ordering on worlds and world morphisms is discrete, so continuity only involves the $A \mathrm{w}^{\prime}\left(u \cdot a, u . a^{\prime}\right)$ argument.

The following proposition is proved using a pullback of the form ${ }_{v}^{u} \diamond_{v^{\prime}}^{u}$.

Proposition 4.2. If $A$ is a pullback-preserving $S V F, u: \mathrm{w} \rightarrow \mathrm{w}^{\prime}$ and $a, a^{\prime} \in A \mathrm{w}$, there is a continuous function $A \mathrm{w}^{\prime}\left(u \cdot a, u . a^{\prime}\right) \rightarrow A \mathrm{w}\left(a, a^{\prime}\right)$. Moreover, the "common ancestor" a of a and $a^{\prime}$ is unique up to $\sim$.

All the SVFs that we define in this paper will turn out to be pullback-preserving. However, for the results described in this paper pullback preservation is not needed. Thus, we will not use it any further, but note that there is always the option to require that property should the need arise subsequently.

Morphisms between functors are natural transformations in the usual sense; they serve to interpret terms with variables and functions. In more explicit terms, a morphism from SVF $A$ to $\mathrm{SVF} B$ is an equivalence class of pairs $e=\left(e_{0}, e_{1}\right)$ where $e_{0}$ and $e_{1}$ are continuous functions of the following types:

$$
\begin{aligned}
& e_{0}: \Pi \mathrm{w} . A \mathrm{w} \rightarrow B \mathrm{w} \\
& e_{1}: \Pi \mathrm{w} . \Pi \mathrm{w}^{\prime} . \Pi x: \mathrm{w} \rightarrow \mathrm{w}^{\prime} . \Pi a \in A \mathrm{w} . \Pi a^{\prime} \in A \mathrm{w}^{\prime} . A \mathrm{w}^{\prime}\left(x . a, a^{\prime}\right) \rightarrow B \mathrm{w}^{\prime}\left(x . e_{0}(a), e_{0}\left(a^{\prime}\right)\right)
\end{aligned}
$$

Again, the requirements for continuity are simplified by the discrete ordering on worlds.

Two morphisms $e=\left(e_{0}, e_{1}\right), e^{\prime}=\left(e_{0}^{\prime}, e_{1}^{\prime}\right)$ are identified if there is a continuous function:

$$
\mu: \Pi w . \Pi a \in A \mathrm{w} . B \mathrm{w}\left(e(a), e^{\prime}(a)\right)
$$

where as in the case of setoids, we omit subscripts where appropriate. These morphisms compose in the obvious way and so the SVFs and morphisms between them form a category.

\section{INSTANCES OF SETOID-VALUED FUNCTORS}

We now describe some concrete functors that will allow us to interpret types of the $v$-calculus as SVFs. The simplest one endows any predomain with the structure of an SVF where the equality is proof-irrelevant and coincides with standard equality. The second one generalises the function space of setoids and is used to interpret function types. The third one is used to model dynamic allocation and is the only one that introduces proper proof-relevance. 
5.1. Base types. For each predomain $D$ we can define a constant $\mathrm{SVF}$, denoted $D$ as well, with $D$ w defined as the discrete setoid over $D$ and $D u$ as the identity. These constant SVFs serve as denotations for base types like booleans or integers.

The SVF $N$ of names is given by $N \mathrm{w}=\mathrm{w}$ where $\mathrm{w}$ on the right hand side stands for the discrete setoid over the discrete predomain of names in $\mathrm{w}$, and $N u=u$ for $u: \mathrm{w} \rightarrow \mathrm{w}^{\prime}$. Thus, e.g. $N\{1,2,3\}=\{1,2,3\}$.

5.2. Cartesian closure. The category of SVFs is cartesian closed, which follows from well-known properties of functor categories. The construction of product and function space follows the usual pattern, but we give it here explicitly.

Let $A$ and $B$ be SVFs. The product $A \times B$ is given by taking a pointwise product of setoids. For the sake of completeness, we note that $(A \times B) \mathrm{w}=A \mathrm{w} \times B \mathrm{w}$ (product predomain) and $(A \times$ $B) \mathrm{w}\left((a, b),\left(a^{\prime}, b^{\prime}\right)\right)=A \mathrm{w}\left(a, a^{\prime}\right) \times B \mathrm{w}\left(b, b^{\prime}\right)$. This defines a cartesian product on the category of SVFs. More generally, we can define the indexed product $\prod_{i \in I} A_{i}$ of a family $\left(A_{i}\right)_{i}$ of SVFs. We write 1 for the empty indexed product and () for the only element of $1 \mathrm{w}$. Note that 1 is the terminal object in the category of SVFs.

The function space $A \Rightarrow B$ is the SVF given as follows. $|(A \Rightarrow B) w|$ contains pairs $\left(f_{0}, f_{1}\right)$ where $f_{0}(u) \in\left|A \mathrm{w}_{1} \Rightarrow B \mathrm{w}_{1}\right|$ for each $\mathrm{w}_{1}$ and $u: \mathrm{w} \rightarrow \mathrm{w}_{1}$. If $u: \mathrm{w} \rightarrow \mathrm{w}_{1}$ and $v: \mathrm{w}_{1} \rightarrow \mathrm{w}_{2}$ then

$$
f_{1}(u, v) \in\left(A \mathrm{w}_{1} \Rightarrow B \mathrm{w}_{2}\right)\left(\left[A v \Rightarrow B \mathrm{w}_{2}\right] f_{0}(v u),\left[A \mathrm{w}_{1} \Rightarrow B v\right] f_{0}(u)\right)
$$

where

$$
\begin{aligned}
& {\left[A v \Rightarrow B \mathrm{w}_{2}\right]:\left(A \mathrm{w}_{2} \Rightarrow B \mathrm{w}_{2}\right) \rightarrow\left(A \mathrm{w}_{1} \Rightarrow B \mathrm{w}_{2}\right)} \\
& {\left[A \mathrm{w}_{1} \Rightarrow B v\right]:\left(A \mathrm{w}_{1} \Rightarrow B \mathrm{w}_{1}\right) \rightarrow\left(A \mathrm{w}_{1} \Rightarrow B \mathrm{w}_{2}\right)}
\end{aligned}
$$

are the obvious composition morphisms.

A proof in $(A \Rightarrow B) \mathrm{w}\left(\left(f_{0}, f_{1}\right),\left(f_{0}^{\prime}, f_{1}^{\prime}\right)\right)$ is a function $g$ that for each $u: \mathrm{w} \rightarrow \mathrm{w}_{1}$ yields a proof $g(u) \in\left(A \mathrm{w}_{1} \Rightarrow B \mathrm{w}_{1}\right)\left(f_{0}(u), f_{0}^{\prime}(u)\right)$.

The order on objects and proofs is pointwise as usual. The following is now clear from the definitions.

Proposition 5.1. The category of SVFs is cartesian closed.

We remark that cartesian closure of the category of SVFs is an instance of the general results (see [nLa]) that if $\mathcal{D}$ is cartesian closed and complete, then so is $\mathcal{D}^{C}$ for any category $C$. Here be $\mathcal{D}$ is the category of setoids described in Section 2.

Definition 5.2. An SVF $D$ is pointed if $D \mathrm{w}$ is pointed for each $w$ and the transition maps $D u: D \mathrm{w} \rightarrow$ $D \mathrm{w}_{1}$ for $u: \mathrm{w} \rightarrow \mathrm{w}_{1}$ are strict.

Theorem 5.3. If $D$ is a pointed $S V F$ then there exists a morphism $Y:(D \Rightarrow D) \rightarrow D$ satisfying the equations from Theorem 2.3 understood relative to the cartesian closed structure of the category of SVFs.

Proof. The fixpoint combinator on the level of SVFs is defined pointwise. Given world $\mathrm{w}$ and $\left(f_{0}, f_{1}\right) \in(D \Rightarrow D)$ w we define

$$
Y \mathrm{w}\left(f_{0}, f_{1}\right)=Y\left(f_{0}\left(i d_{\mathrm{w}}\right)\right)
$$

where $Y$ is the setoid fixpoint combinator from Theorem 2.3. The translation of proofs is obvious. We need to show that this defines a natural transformation. So, let $u: \mathrm{w} \rightarrow \mathrm{w}_{1}$ and $\left(f_{0}, f_{1}\right) \in(D \Rightarrow D) \mathrm{w}$. Put $f:=f_{0}\left(i d_{\mathrm{w}}\right)$ and $g:=f_{0}(u)$. We need to construct a proof that $D u(Y(f)) \sim Y(g)$. Now, $f_{1}$ furnishes a proof of $(D u) f=g$ and $D u$ is strict by assumption on $D$ so that "Uniformity" furnishes the desired proof. 
The laws from Theorem 2.3 can be directly inherited.

Definition 5.4. An SVF $A$ is discrete if $A \mathrm{w}$ is a discrete setoid for every world w.

The constructions presented so far only yield discrete SVFs, i.e., proof relevance is merely propagated, but never actually created. This is not so for the next operator on SVFs, which is to model dynamic allocation.

\section{Dynamic Allocation Monad}

Before we define the dynamic allocation monad we recall Stark's [Sta94] definition of a dynamic allocation monad for the category of set-valued functors on the category of worlds. For set-valued functor $A$, Stark defines a set-valued functor $T A$ by $T A \mathrm{w}=\left\{\left(\mathrm{w}_{1}, a\right) \mid \mathrm{w} \subseteq \mathrm{w}_{1}, a \in A \mathrm{w}_{1}\right\} / \sim$ where $\left(\mathrm{w}_{1}, a\right) \sim\left(\mathrm{w}_{1}^{\prime}, a^{\prime}\right)$ iff there exist maps $x: \mathrm{w}_{1} \rightarrow \overline{\mathrm{w}}, x^{\prime}: \mathrm{w}_{1}^{\prime} \rightarrow \overline{\mathrm{w}}$ for some $\overline{\mathrm{w}}$ satisfying $x . i=x^{\prime} . i^{\prime}$ and $x . a=x^{\prime} \cdot a^{\prime}$ where $i: \mathrm{w} \hookrightarrow \mathrm{w}_{1}$ and $i^{\prime}: \mathrm{w} \hookrightarrow \mathrm{w}_{1}^{\prime}$ are the inclusion maps.

Our dynamic allocation monad for SVFs essentially mimics this definition, the difference being that the maps $i, i^{\prime}$ witnessing equivalence of elements now become proofs of $\sim$-equality. Additionally, our definition is based on predomains and involves a bottom element for recursion.

\subsection{Definition of the monad. Let $A$ be an SVF. We put}

$$
|T A \mathrm{w}|=\left\{\left(\mathrm{w}_{1}, a\right) \mid \mathrm{w} \subseteq \mathrm{w}_{1} \wedge a \in A \mathrm{w}_{1}\right\}_{\perp}
$$

Thus, a non-bottom element of $T A \mathrm{w}$ consists of an extension of $\mathrm{w}$ together with an element of $A$ taken at that extension. Note that the extension is not existentially quantified, but an inherent part of the element.

The ordering is given by $\left(\mathrm{w}_{1}, a\right) \leq\left(\mathrm{w}_{1}^{\prime}, a^{\prime}\right)$ if $\mathrm{w}_{1}=\mathrm{w}_{1}^{\prime}$ and $a \leq a^{\prime}$ in $A \mathrm{w}_{1}$ and of course, $\perp$ is the least element of $T A \mathrm{w}$.

The proofs are defined as follows. First, $\operatorname{TAw}(\perp, \perp)=\{\perp\}$ and second, the elements of $T A \mathrm{w}\left(\left(\mathrm{w}_{1}, a\right),\left(\mathrm{w}_{1}^{\prime}, a^{\prime}\right)\right)$ are triples $\left(x, x^{\prime}, p\right)$ where $x, x^{\prime}$ complete the inclusions $u: \mathrm{w} \hookrightarrow \mathrm{w}_{1}$ and $u^{\prime}: \mathrm{w} \hookrightarrow \mathrm{w}_{1}^{\prime}$ to a commuting square

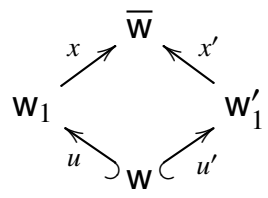

with $\overline{\mathrm{w}}=\operatorname{cod}(x)=\operatorname{cod}\left(x^{\prime}\right)$. The third component $p$ then is a proof that $a$ and $a^{\prime}$ are equal when transported to $\overline{\mathrm{w}}$, formally, $p \in A \overline{\mathrm{w}}\left(x \cdot a, x^{\prime} \cdot a^{\prime}\right)$. The ordering is again discrete in $x, x^{\prime}$ and inherited from $A$ in $p$. Formally, $\left(\left(\mathrm{w}_{1}, a\right),\left(\mathrm{w}_{1}^{\prime}, a^{\prime}\right),\left(x, x^{\prime}, p\right)\right) \leq\left(\left(\mathrm{w}_{1}, b\right),\left(\mathrm{w}_{1}^{\prime}, b^{\prime}\right),\left(x, x^{\prime}, q\right)\right)$ when $\left(x . a, x^{\prime} \cdot a^{\prime}, p\right) \leq\left(x . b, x^{\prime} . b, q\right)$ in $A \operatorname{cod}(x)$ and of course $(\perp, \perp, \perp)$ is the least element. No $\leq$-relation exists between triples with different mediating co-span. In particular, in an ascending chain of proofs the witnessing spans are always the same, which is the intuitive reason why they can be patched together to form a supremum.

Consider, for example, that $w=\{0\}, w_{1}=\{0,1,2\}, w_{1}^{\prime}=\{0,2,3\}$. Then, both $c=\left(w_{1},(0,2)\right)$ and $\mathrm{c}^{\prime}=\left(\mathrm{w}_{1}^{\prime},(0,3)\right)$ are elements of $T(N \times N) \mathrm{w}$, and $\left(x, x^{\prime}, p\right) \in T(N \times N) \mathrm{w}\left(\mathrm{c}, \mathrm{c}^{\prime}\right)$ is a proof that the two are equal where $x: \mathrm{w}_{1} \rightarrow \overline{\mathrm{w}}=\{0,1,2,3\}$ sends $0 \mapsto 0,1 \mapsto 1,2 \mapsto 2$ and $x^{\prime}: \mathrm{w}_{1}^{\prime} \rightarrow \overline{\mathrm{w}}$ sends $0 \mapsto 0,2 \mapsto 3,3 \mapsto 2$. The proof $p$ is the canonical proof by reflexivity. Note that, in this case, the order relation is trivial. It becomes more interesting when the type of values $A$ is a function space. 
Next, we define the morphism part. Assume that $u: \mathbf{w} \rightarrow \mathrm{q}$ is a morphism in $\mathbf{W}$. We want to construct a morphism $A u: T A \mathrm{w} \rightarrow T A \mathrm{q}$ in $\operatorname{Std}$. So let $\left(\mathrm{w}_{1}, a\right) \in T A \mathrm{w}$ and $i: \mathrm{w} \hookrightarrow \mathrm{w}_{1}$ be the inclusion. We complete the span $i, u$ to a minimal pullback

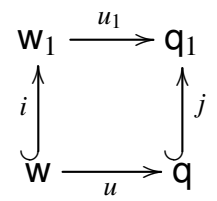

with $j$ an inclusion as indicated. We then define $T A u\left(\mathrm{w}_{1}, a\right)=\left(\mathrm{q}_{1}, u_{1} . a\right)$. We assume a function that returns such completions to minimal pullbacks in some chosen way. The particular choice is unimportant.

Picking up the previous example and letting $u: \mathrm{w} \rightarrow \mathrm{q}=\{0,1\}$ be $0 \mapsto 1$ then a possible completion to a minimal pullback would be

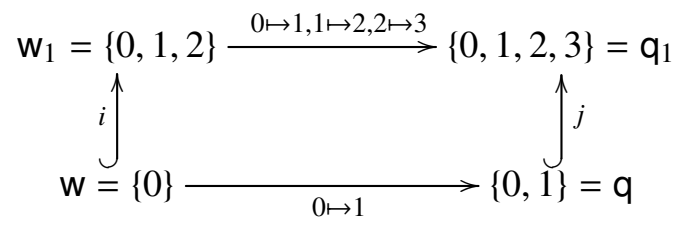

Note that the following square where the additional name 1 in q is identified with a name already existing in $\mathrm{w}_{1}$ is not a pullback

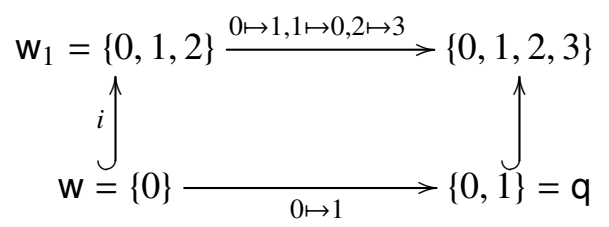

Adding extra garbage into $\mathrm{q}_{1}$ like so would result in a pullback that is not minimal.

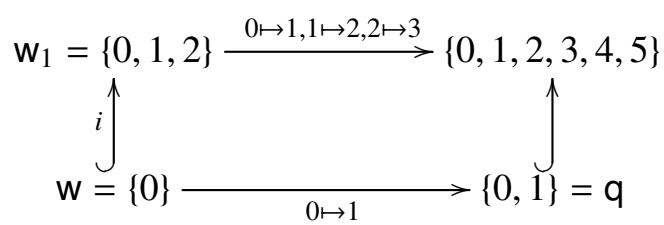

If $\left(x, x^{\prime}, p\right)$ is a proof of $\left(\mathrm{w}_{1}, a\right) \sim\left(\mathrm{w}_{1}^{\prime}, a^{\prime}\right)$ then we obtain a proof, $\left(\mathrm{q}_{1}^{\prime}, u_{1}^{\prime} \cdot a^{\prime}\right)$, that $T A u\left(\mathrm{w}_{1}, a\right) \sim$ $T A u\left(\mathrm{w}_{1}^{\prime}, a^{\prime}\right)$ as follows. We first complete the span $x i, u$ to a minimal pullback with apex $\overline{\mathrm{q}}$ and upper arrow $\bar{u}: \operatorname{cod}(x)=\overline{\mathrm{w}} \rightarrow \overline{\mathrm{q}}$. Now minimality of the pullbacks apexed at $\mathrm{q}_{1}$ and $\mathrm{q}_{1}^{\prime}$ furnishes morphisms $y: \mathrm{q}_{1} \rightarrow \overline{\mathrm{q}}$ and $y^{\prime}: \mathrm{q}_{1}^{\prime} \rightarrow \overline{\mathrm{q}}$ so that $y j=y^{\prime} j^{\prime}$ (where $j^{\prime}: \mathrm{q} \hookrightarrow \mathrm{q}_{1}^{\prime}$ ). We then have $\left(y, y^{\prime}, \bar{u} . p\right): T A u\left(\mathrm{w}_{1}, a\right) \sim T A u\left(\mathrm{w}_{1}^{\prime}, a^{\prime}\right)$ as required. This shows that the passage $\left(\mathrm{w}_{1}, a\right) \mapsto\left(\mathrm{w}_{1}^{\prime}, a^{\prime}\right)$ actually does define a morphism of setoids.

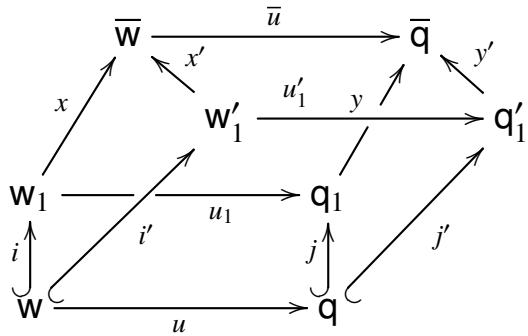


The functor laws amount to similar constructions of $\sim$-witnesses and are left to the reader. The following is direct from the definitions.

Proposition 6.1. $T$ is a strong monad on the category of $S V F s$. The unit sends $v \in A \mathrm{w}$ to $(\mathrm{w}, \mathrm{v}) \in$ $(T A) \mathrm{w}$. The multiplication sends $\left(\mathrm{w}_{1},\left(\mathrm{w}_{2}, v\right)\right) \in(T T A) \mathrm{w}$ to $\left(\mathrm{w}_{2}, v\right) \in T A \mathrm{w}$. The strength map sends $\left(a,\left(\mathrm{w}_{1}, b\right)\right) \in(A \times T B) \mathrm{w}$ to $\left(\mathrm{w}_{1},(a . i, b)\right)$ where $i: \mathrm{w} \hookrightarrow \mathrm{w}_{1}$.

Notice that if we had taken any arbitrary commuting square, like the one shown in Equation 6.1, then preservation of proofs could not be guaranteed because names in extensions would be captured in an arbitrary way. Requiring minimality, on the other hand, is merely a technical convenience.

6.2. Comparison with cpo-valued functors. The flawed attempt at defining a dynamic allocation monad for FM-domains discussed by Shinwell [Shi04] and mentioned in the introduction can be reformulated in terms of cpo-valued functors and further highlights the importance of proof-relevant equality.

Given a cpo-valued functor $A$ one may construct a poset-valued functor $T_{s p} A$ which has for underlying set equivalence classes of pairs $\left(\mathrm{w}_{1}, a\right)$ with $\mathrm{w} \subseteq \mathrm{w}_{1}$ and $a \in A \mathrm{w}_{1}$. As in Stark's definition above, we have a $\left(\mathrm{w}_{1}, a\right) \sim\left(\mathrm{w}_{1}^{\prime}, a^{\prime}\right)$ if there are morphisms $x, x^{\prime}$ such that $x i=x^{\prime} i^{\prime}$ and $x . a=x^{\prime} \cdot a^{\prime}$ where $i: \mathrm{w} \rightarrow \mathrm{w}_{1}, i^{\prime}: \mathrm{w} \rightarrow \mathrm{w}_{1}^{\prime}$ are the inclusions. As for the ordering, the only reasonable choice is to decree that on representatives $\left(\mathrm{w}_{1}, a\right) \leq\left(\mathrm{w}_{1}^{\prime}, a^{\prime}\right)$ if $x . a \leq x^{\prime} . a^{\prime}$ for some co-span $x, x^{\prime}$ with $x i=x^{\prime} i^{\prime}$ where $i, i^{\prime}$ are the inclusions as above. However, while this defines a partial order it is not clear why it should have suprema of ascending chains because the witnessing spans might not match up so that they can be pasted to a witnessing span for the limit of the chain. Indeed, Shinwell's thesis [Shi04] contains a concrete counterexample, which is due to Pitts.

In our notation, Pitts's counterexample takes the following form. Define the cpo-valued functor $A$ by $A \mathrm{w}:=(\mathcal{P}(\mathrm{w}), \subseteq)$. So the elements of $A \mathrm{w}$ are subsets of $\mathrm{w}$ ordered by inclusion, hence a finite cpo. Let us now examine $T_{s p} A$. An element of $T_{s p} A \mathrm{w}$ is an $\sim$-equivalence class of pairs $\left(\mathrm{w}_{1}, U\right)$ where $U \subseteq \mathrm{w}_{1}, \mathrm{w} \subseteq \mathrm{w}_{1}$. Furthermore, $\left(\mathrm{w}_{1}, U\right) \sim\left(\mathrm{w}_{1}^{\prime}, U^{\prime}\right)$ whenever $U=U^{\prime}$ and the ordering $\leq$ on $T_{s p} A \mathrm{w}$ is $\left(\mathrm{w}_{1}, U\right) \leq\left(\mathrm{w}_{1}^{\prime}, U^{\prime}\right)$ whenever $U \subseteq U^{\prime}$. Let $t_{n}$ be the equivalence class of $(\{0, \ldots, n-1\},\{0, \ldots, n-1\})$. We have $t_{n} \in T_{s p} A \emptyset$ for all $n$ and $t_{n} \leq t_{m} \Longleftrightarrow n \leq m$. From this it is clear that the ascending chain $t_{0} \leq t_{1} \leq \cdots$ does not have a least upper bound in $T_{s p} A \emptyset$ for if $\left(\mathrm{w}_{1}, U\right)$ were such an upper bound then $|U| \geq n$ would have to hold for all $n$.

The transition to proof relevance that we have made allows us to define the order on representatives as we have done and thus to bypass these difficulties. We view $A$ above as an SVF with underlying cpo $A \mathrm{w}=\mathrm{w}$ and, trivial, i.e., discrete equality. Now applying our dynamic allocation monad $T$ to $A$ yields the SVF $T A \mathrm{w}$ whose underlying cpo contains in addition to $\perp$, pairs $\left(\mathrm{w}_{1}, U\right)$ where $U \subseteq \mathrm{w}_{1}$ with ordering $\left(\mathrm{w}_{1}, U\right) \leq\left(\mathrm{w}_{1}^{\prime}, U^{\prime}\right)$ if $\mathrm{w}_{1}=\mathrm{w}_{1}^{\prime}$ and $U \subseteq U^{\prime}$. A proof that an element $\left(\mathrm{w}_{1}, U\right)$ is equal to the element $\left(\mathrm{w}_{1}^{\prime}, U^{\prime}\right)$ is given by a triple $\left(\mathrm{w}_{2}, u, u^{\prime}\right)$ such that $u: \mathrm{w}_{1} \rightarrow \mathrm{w}_{2}$ and $u^{\prime}: \mathrm{w}_{1}^{\prime} \rightarrow \mathrm{w}_{2}$ and moreover $u(U)=u^{\prime}\left(U^{\prime}\right)$. The ordering in these proofs is the discrete one. Now the sequence shown above is not an ascending chain and thus is no longer a counter-example to completeness.

\section{Observational Equivalence and Fundamental Lemma}

We now construct the machinery that connects the concrete language with the denotational machinery introduced in Section 1. The semantics of types, written using $\mathbb{I} \cdot \mathbb{1}$, as SVFs is defined inductively as follows:

- For basic types $\llbracket \tau \rrbracket$ is the corresponding discrete SVF. 
- $\llbracket \tau \rightarrow \tau^{\prime} \rrbracket$ is defined as the function space $\llbracket \tau \rrbracket \rightarrow T \llbracket \tau^{\prime} \rrbracket$, where $T$ is the dynamic allocation monad.

- For typing context $\Gamma$ we define $\llbracket \Gamma \rrbracket$ as the indexed product of SVFs $\prod_{x \in \operatorname{dom}(\Gamma)} \llbracket \Gamma(x) \rrbracket$.

To each term in context $\Gamma \vdash e: \tau$ we can associate a morphism $\llbracket e \rrbracket$ from $\llbracket \Gamma \rrbracket$ to $T \llbracket \tau \rrbracket$ by interpreting the syntax in the category of SVFs using cartesian closure, the fixpoint combinator, and the fact that $T$ is a strong monad. We omit most of the straightforward but perhaps slightly tedious definition and only give the clauses for "new" and "let" here:

$$
\llbracket \text { new } \rrbracket \mathrm{w}=(\mathrm{w} \cup\{n+1\}, n+1)
$$

where $n=\max (\mathrm{w})$ and $\max (\mathrm{w})=\max (\{n \mid n \in \mathrm{w}\})$, i.e., the greatest number in the world $\mathrm{w}$.

If $f_{1}: \llbracket \Gamma \rrbracket \rightarrow T \llbracket \tau_{1} \rrbracket$ and $f_{2}: \llbracket \Gamma, x: \tau_{1} \rrbracket \rightarrow T \llbracket \tau_{2} \rrbracket$ are the denotations of $\Gamma \vdash e_{1}: \tau_{1}$ and $\Gamma, x: \tau_{2} \vdash e_{2}: \tau_{2}$ then the interpretation of let $x \Leftarrow e_{1}$ in $e_{2}$ is the morphism $f: \llbracket \Gamma \rrbracket \rightarrow T \llbracket \tau_{2} \rrbracket$ given by

$$
f=\mu \circ T f_{2} \circ \sigma \circ\left\langle i d_{\Gamma}, f_{1}\right\rangle
$$

where $\mu$ is the monad multiplication, $\sigma$ is the monad strength and where we have made the simplifying assumption that $\llbracket \Gamma, x: \tau \rrbracket=\llbracket \Gamma \rrbracket \times \llbracket \tau \rrbracket$. Assuming that $f_{1}$ and $f_{2}$ now stand for the first components of concrete representatives of these morphisms, one particular concrete representative of this morphism (now also denoted $f$ ) satisfies:

$$
f \mathrm{w}(\gamma)=f_{2}(i . \gamma, a) \text {, where } i \text { is the inclusion } \mathrm{w} \hookrightarrow \mathrm{w}_{1} \text { and } f_{1} \mathrm{w}(\gamma)=\left(\mathrm{w}_{1}, a\right) .
$$

Our aim is now to relate these morphisms to the computational interpretation $\llbracket e \rrbracket$.

Definition 7.1. For each type $\tau$ and world $\mathrm{w}$ we define two relations; the relation $\Vdash_{\mathrm{w}}^{\tau} \subseteq \| \tau \rrbracket \times \llbracket \tau \rrbracket \mathrm{w}$ and $\Vdash_{\mathrm{w}}^{T \tau} \subseteq\left(\mathbb{N} \rightarrow(\mathbb{N} \times\|\tau\|)_{\perp}\right) \times T \llbracket \tau \rrbracket \mathrm{w}$ by the following clauses.

$$
\begin{aligned}
& b \Vdash_{\mathrm{w}}^{\text {bool }} \mathrm{b} \Longleftrightarrow b=\mathrm{b} \\
& i \Vdash_{\mathrm{w}}^{\text {int } \mathrm{i}} \Longleftrightarrow i=\mathrm{i} \\
& l \mathbb{1}_{\mathrm{w}}^{\text {name }} k \Longleftrightarrow l=k \\
& f \Vdash_{\mathrm{w}}^{\tau \rightarrow \tau^{\prime}} g \Longleftrightarrow \forall \mathrm{w}_{1} \supseteq \mathrm{w} \cdot \forall v \cdot \forall \mathrm{v} \cdot v \Vdash_{\mathrm{w}_{1}}^{\tau} \mathrm{v} \Rightarrow f(v) \Vdash_{\mathrm{w}_{1}}^{T \tau^{\prime}} g_{0}\left(\mathrm{w} \hookrightarrow \mathrm{w}_{1}, \mathrm{v}\right) \\
& c \Vdash_{\mathrm{w}}^{T \tau} \mathrm{c} \Longleftrightarrow \quad[c(\max (\mathrm{w})+1)=\perp \Leftrightarrow \mathrm{c}=\perp] \wedge \\
&
\end{aligned}
$$

Notice that $T \tau$ is not part of the syntax, but $T$ is a marker to distinguish the two relations defined above.

The following lemma states that the realizability relation is stable with respect to enlargement of worlds. It is needed for the "fundamental lemma" 7.3.

Lemma 7.2. Let $\tau$ be a type. If $u: \mathrm{w} \hookrightarrow \mathrm{w}_{1}$ is an inclusion as indicated and $v \Vdash_{\mathrm{w}}^{\tau} \mathrm{v}$ then $v \Vdash_{\mathrm{w}_{1}}^{\tau} u . \mathrm{v}$, too.

The proof is by a straightforward induction on types. Note, however, that the restriction to inclusions is important for the cases of function type and the type name. We extend $\Vdash$ to typing contexts by putting

for $\eta \in \llbracket \Gamma \rrbracket$ and $\gamma \in \llbracket \Gamma \rrbracket$.

$$
\eta \Vdash_{\mathrm{w}}^{\Gamma} \gamma \Longleftrightarrow \forall x \in \operatorname{dom}(\Gamma) \cdot \eta(x) \Vdash_{\mathrm{w}}^{\Gamma(x)} \gamma(x)
$$

Theorem 7.3 (Fundamental lemma). Let $\Gamma \vdash e: \tau$ be a well typed term. There exists a representative $\left(\mathrm{c},{ }_{-}\right)$of the equivalence class $\llbracket e \rrbracket$ at world $\mathrm{w}$ such that if $\eta \Vdash_{\mathrm{w}}^{\Gamma} \gamma$ then $\| e \rrbracket \eta \Vdash_{\mathrm{w}}^{T \tau} \mathrm{c}(\gamma)$. 
Proof. By induction on typing rules. We always chose for the representative the one given as witness in the definition of $\llbracket e \rrbracket$. Most of the cases are straightforward. For illustration we show new and let : As for new, we pick the representative $c$ that at world $w$ returns $(w \cup\{\max (w)+1\}, \max (w))$. Now, with $c=\|$ new $\|$, we have $c(\max (\mathrm{w}))=(\max (\mathrm{w})+1, \max (\mathrm{w}))$ and $c \mathbb{}_{\mathrm{w}}^{T N} \mathrm{c}$ holds, since $\max (w \cup\{\max (w)+1\})=\max (w)+1$.

Next, assume that $\Gamma \vdash$ let $x \Leftarrow e_{1}$ in $e_{2}: \tau_{2}$, where $\Gamma \vdash e_{1}: \tau_{1}$ and $\Gamma, x: \tau_{1} \vdash e_{2}: \tau_{2}$. Choose, according to the induction hypothesis appropriate representatives $c_{1}$ of $\llbracket e_{1} \rrbracket$ and $c_{2}$ of $\llbracket e_{2} \rrbracket$. If $\eta \Vdash_{\mathrm{w}}^{\Gamma} \gamma$ for some initial world $\mathrm{w}$ then we have (H1) $\left\|e_{1}\right\| \eta \Vdash_{\mathrm{w}}^{T \tau_{1}} \mathrm{c}_{1}(\gamma)$. If $\left\|e_{1}\right\| \eta(\max (\mathrm{w})+1)=\perp$ then $\mathrm{c}_{1}(\gamma)=\perp$, too, and the same goes for the interpretation of the entire let-construct. So suppose that $\| e_{1} \rrbracket \eta(\max (\mathrm{w})+1)=\left(n_{1}, v\right)$. By $(\mathrm{H} 1)$, we must then have $\mathrm{c}_{1}(\gamma)=\left(\mathrm{w}_{1}, \mathrm{v}\right)$ where $\mathbf{w} \subseteq \mathrm{w}_{1}$ and $n_{1}=\max \left(\mathrm{w}_{1}\right)+1$ and $v \Vdash_{\mathrm{w}_{1}}^{\tau_{1}} \mathrm{v}$.

By Lemma 7.2 we then have $\eta \mathbb{w}_{\mathrm{w}_{1}}^{\Gamma} i . \gamma$ where $i: \mathrm{w} \hookrightarrow \mathrm{w}_{1}$. Thus, by the induction hypothesis, we get (H2) $\llbracket e_{2} \rrbracket(\eta[x \mapsto v]) \Vdash_{\mathrm{w}_{1}}^{T \tau_{2}} \mathrm{c}_{2}(i . \gamma[x \mapsto \mathrm{v}])$. Thus, putting $\mathrm{c}(\gamma)=\mathrm{c}_{2}(i . \gamma, \mathrm{v})$ furnishes the required representative of $\llbracket$ let $x \Leftarrow e_{1}$ in $e_{2} \rrbracket(\mathrm{w})$.

Remark 7.4. Note that the particular choice of representative matters here. For example, if $c_{0} w=$ $(\mathrm{w} \uplus\{\max (w)+1, \max (w)+2\}, \max (w)+1)$ then there exists $\mathrm{c}_{1}$ such that $\left(\mathrm{c}_{0}, \mathrm{c}_{1}\right): 1 \rightarrow T N$ and $\left(\mathrm{c}_{0}, \mathrm{c}_{1}\right)$ and $\llbracket$ new $\rrbracket$ are equal qua morphisms of SVFs. Yet, $\|$ new $\| \nVdash_{\mathrm{w}}^{T N} \mathrm{c}_{0}$.

It would have been an option to refrain from the identification of $\sim$-related morphisms. The formulation of the Fundamental Lemma would then have become slightly easier as we would have defined $\llbracket e \rrbracket$ so as to yield the required witnesses directly. On the other hand, the equational properties of the so obtained category would be quite weak and in particular cartesian closure, monad laws, functor laws, etc would only hold up to $\sim$. This again would not really be a problem but prevent the use of standard category-theoretic terminology.

\subsection{Observational Equivalence.}

Definition 7.5. Let $\tau$ be a type. We define an observation of type $\tau$ as a closed term $\vdash o: \tau \rightarrow$ bool. Two values $v, v^{\prime} \in \llbracket \tau \|$ are observationally equivalent at type $\tau$ if for all observations $o$ of type $\tau$ one has that $\llbracket o \rrbracket(v)(0)$ is defined iff $\llbracket o \|\left(v^{\prime}\right)(0)$ is defined and when $\llbracket o \rrbracket(v)(0)=\left(n_{1}, v_{1}\right)$ and $\llbracket o \rrbracket\left(v^{\prime}\right)(0)=\left(n_{1}^{\prime}, v_{1}^{\prime}\right)$ then $v_{1}=v_{1}^{\prime}$.

Note that observational equivalence is a congruence since an observation can be extended by any englobing context. We also note that observational equivalence is the coarsest reasonable congruence.

We now show how the proof-relevant semantics can be used to deduce observational equivalences.

Theorem 7.6 (Observational equivalence). If $\tau$ is a type and $v \Vdash_{\emptyset}^{\tau} e$ and $v^{\prime} \Vdash_{\emptyset}^{\tau} e^{\prime}$ with $e \sim e^{\prime}$ in $\llbracket \tau \rrbracket \emptyset$ then $v$ and $v^{\prime}$ are observationally equivalent at type $\tau$.

Proof. Let $o$ be an observation at type $\tau$. By the Fundamental Lemma (Theorem 7.3) we have $\llbracket o \rrbracket \Vdash_{\emptyset}^{\tau \rightarrow \text { bool } \llbracket o \rrbracket . ~}$

Now, since $e \sim e^{\prime}$ we also have $\llbracket o \rrbracket(e) \sim \llbracket o \rrbracket\left(e^{\prime}\right)$ and, of course, $\llbracket o \rrbracket(v) \mathbb{}_{\emptyset}^{T \text { bool }} \llbracket o \rrbracket(e)$ and $\llbracket o \rrbracket\left(v^{\prime}\right) \Vdash_{\emptyset}^{T \text { bool }} \llbracket o \rrbracket\left(e^{\prime}\right)$.

Knowing $\llbracket o \rrbracket(e) \sim \llbracket o \rrbracket\left(e^{\prime}\right)^{1}$, there are two cases. If $\llbracket o \rrbracket(e)(0)$ and $\llbracket o \rrbracket\left(e^{\prime}\right)(0)$ both diverge, then the same is true for $\llbracket o \rrbracket(v)(0)$ and $\llbracket o \rrbracket\left(v^{\prime}\right)(0)$ by definition of $\|^{T \text { bool }}$. Alternatively, if $\llbracket o \rrbracket(e)(0)=$ $\left({ }_{-},{ }_{-}, b,,_{-}\right)$and $\llbracket o \rrbracket\left(e^{\prime}\right)(0)=\left({ }_{-},{ }_{-}, b^{\prime},{ }_{-}\right)$for booleans $b, b^{\prime}$ then, by definition of $\sim$ at $T \llbracket$ bool $\rrbracket$ we get

\footnotetext{
${ }^{1}$ More precisely, we are using the representative of the equivalence class given by Theorem 7.3.
} 
$b=b^{\prime}$ and, again by definition of $\Vdash^{T \text { bool }}$, this then implies that $\left.\llbracket o \rrbracket(v)(0)={ }_{(}, b\right)$ and $\llbracket o \rrbracket(v)(0)=$ $\left(-, b^{\prime}\right)$ with $b=b^{\prime}$, hence the claim.

\section{Direct-Style Proofs}

We now have enough machinery to provide direct-style proofs for equivalences involving name generation.

If $\Gamma \vdash e: \tau$ and $\Gamma \vdash e^{\prime}: \tau$, we say the equation $\Gamma \vdash e=e^{\prime}: \tau$ is semantically sound if $\llbracket e \rrbracket=\llbracket e^{\prime} \rrbracket$ are equal morphisms from $\llbracket \Gamma \rrbracket$ to $\llbracket \tau \rrbracket$. If $v=v^{\prime}$ can be derived by sound equations and congruence rules, then $\llbracket v \rrbracket$ and $\llbracket v^{\prime} \rrbracket$ are equivalent by Theorem 7.6. We omit the formal definition of such derivations using an equational theory. We refer to [BHN14] for details on how this could be set up.

From the categorical properties of setoids the soundness of $\beta, \eta$, fixpoint unrolling and similar equations is obvious. We now demonstrate the soundness of the more interesting equations involving name generation.

8.1. Drop equation. We start with the following equation, which eliminates a dummy allocation:

$$
\text { (let } x \Leftarrow \text { new in } e \text { ) }=e, \quad \text { provided } x \text { is not free in } e \text {. }
$$

Formally we have $\Gamma \vdash e: \tau$ and, writing $c$ for the LHS of the above equation, and $c^{\prime}$ for the RHS, the equation reads $\Gamma \vdash c=c^{\prime}: \tau$. We have $\llbracket c^{\prime} \rrbracket \mathrm{w}(\gamma)=\left(\mathrm{w}_{1}, v\right)$ for some extension $\mathrm{w}_{1}$ of $\mathrm{w}$ and $v: \llbracket \tau \rrbracket \mathrm{w}_{1}$ and $\llbracket c \rrbracket \mathrm{w}(\gamma)=\left(\mathrm{w}_{2}, i . v\right)$ where $\mathrm{w}_{2}=\mathrm{w}_{1} \cup\left\{\max \left(\mathrm{w}_{1}\right)+1\right\}$ and $i: \mathrm{w}_{1} \hookrightarrow \mathrm{w}_{2}$.

Now it remains to construct a proof of $\left(\mathrm{w}_{1}, v\right) \sim\left(\mathrm{w}_{2}, v\right) \in T A \mathrm{w}$, which should depend continuously on $\gamma$. To that end, we consider the following pullback square, where the annotations above and below the square are just to illustrate in which world the semantic values are:

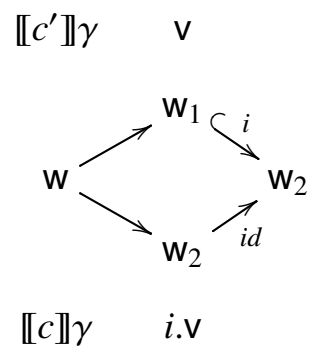

Clearly we have $i . \mathrm{v} \sim i d . i . \mathrm{v}$ and therefore the pullback above is a proof that $\left(\mathrm{w}_{1}, v\right) \sim\left(\mathrm{w}_{2}, v\right) \in T A \mathrm{w}$.

8.2. Swap equation. Let us now consider the following equivalence where the order in which the names are generated is switched:

$$
\text { (let } x \Leftarrow \text { new in let } y \Leftarrow \text { new in } e)=(\text { let } y \Leftarrow \text { new in let } x \Leftarrow \text { new in } e \text { ). }
$$

Again, we write $c$ for the LHS and $c^{\prime}$ for the RHS. Let $I_{1}, I_{2}, l_{1}^{\prime}, l_{2}^{\prime}$ be the concrete locations allocated by the left-hand-side and right-hand-side of the equation. In fact, $l_{1}=\max (w)+1, l_{2}=I_{1}+1$ and $\mathrm{I}_{2}^{\prime}=\mathrm{I}_{1}$ and $\mathrm{I}_{1}^{\prime}=\mathrm{I}_{2}$. We have $\llbracket c \rrbracket \gamma=\left(\mathrm{w}_{2}, v\right)$, where $\llbracket e \rrbracket\left(i . \gamma\left[x \mapsto \mathrm{I}_{1}, y \mapsto \mathrm{I}_{2}\right]\right)=\left(\mathrm{w}_{2}, v\right)$. We also have $\llbracket c^{\prime} \rrbracket \gamma$, where $\llbracket e \rrbracket\left(i^{\prime} \cdot \gamma\left[x \mapsto \mathrm{I}_{2}^{\prime}, y \mapsto \mathrm{I}_{1}^{\prime}\right]\right)=\left(\mathrm{w}_{2}^{\prime}, v^{\prime}\right)$. 
Define $s\left(\mathrm{I}_{1}\right)=\mathrm{I}_{2}^{\prime}, s\left(\mathrm{l}_{2}\right)=\mathrm{l}_{1}^{\prime}$ and $s 1 \mathrm{w}=i d$. Naturality of $\llbracket e \rrbracket$, i.e., $\llbracket e \rrbracket \circ \llbracket \Gamma, x, y \rrbracket s \sim T \llbracket t \rrbracket s \circ \llbracket e \rrbracket$ furnishes a co-span $x, x^{\prime}$ so that $x . s_{2} . v \sim x^{\prime} . v^{\prime}$ and $x t=x^{\prime} u_{1}^{\prime}$ (III). Here $s_{2}, t$ is the completion of the span $s_{1}, u_{1}$ to a minimal pullback as contained in the definition of $T \llbracket \tau \rrbracket s$.

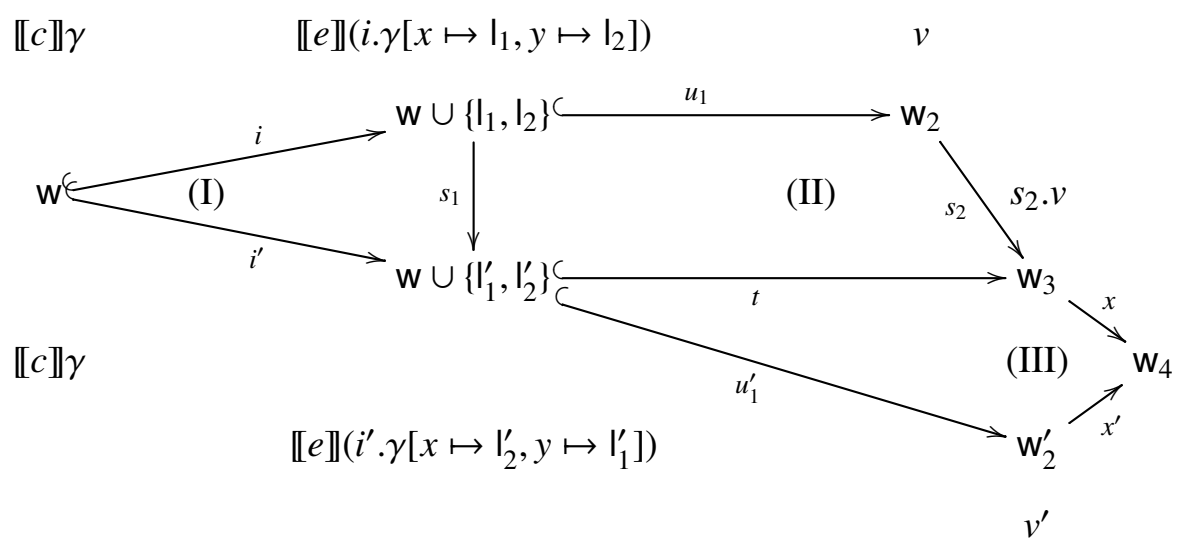

Notice that the square (I) commutes by definition of $s_{1}$; the square (II) commutes because it is a minimal pullback. As a results the entire diagram commutes. $x s_{2} u_{1}$ and $x^{\prime} u_{1}^{\prime}$ is the proof that $\llbracket c \rrbracket \gamma \sim \llbracket c^{\prime} \rrbracket \gamma$.

This is essentially the same proof as given by Stark [Sta94], but now it also works in the presence of recursion.

\section{Proof-ReleVANT PARAMETRic FUnCtors}

The following equation (Stark's "Equivalence 12") cannot be validated in the functor category model and nor is it valid in the category of SVFs.

$$
\text { (let } n \Leftarrow \text { new in fun } x . x=n)=(\text { fun } x \text {.false). }
$$

The above is, nevertheless, a valid contextual equivalence. The intuition is that the name $n$ generated in the left-hand side is never revealed to the context and is therefore distinct from any name that the context might pass in as argument to the function; hence, the function will always return false. To justify this equivalence, Stark constructs a model based on traditional Kripke logical relations. $\mathrm{He}$ also gives a category-theoretic version of that logical relation using so-called parametric functors. In this section, we construct a proof-relevant version of these parametric functors, which will allow us to justify the above equivalence in the presence of recursion and in direct style. In fact, this seems to be the first time that this equivalence has been established in this setting; we are not aware of an earlier extension of parametric functors to recursion.

We also show that the transition to proof relevance makes the induced logical relation transitive, which is generally not the case for ordinary Kripke logical relations.

9.1. Spans of Worlds. We use capital letters $S, S^{\prime}, \ldots$ for spans of worlds. If $S$ is the span $\mathrm{w} \stackrel{u}{\leftarrow} \underline{\mathrm{w}} \stackrel{u^{\prime}}{\rightarrow} \mathrm{w}^{\prime}$ then we use the notations $S: \mathrm{w} \leftrightarrow \mathrm{w}^{\prime}$ and $\mathrm{w}=\operatorname{dom}(S)$ (left domain), $\mathrm{w}^{\prime}=\operatorname{dom}^{\prime}(S)$ (right domain), $\underline{\mathrm{w}}=\operatorname{lop}(S)$ (low point), $u=S . u, u^{\prime}=S . u^{\prime}$. For world $\mathrm{w}$ we denote $r(\mathrm{w}): \mathrm{w} \leftrightarrow \mathrm{w}$ the identity span $\mathrm{w} \stackrel{i d}{\leftarrow} \mathrm{w} \stackrel{\text { id }}{\rightarrow} \mathrm{w}$. If $S: \mathrm{w} \leftrightarrow \mathrm{w}^{\prime}$ then $s(S): \mathrm{w}^{\prime} \leftrightarrow \mathrm{w}$ is given by $\mathrm{w}^{\prime} \stackrel{S . u^{\prime}}{\leftarrow} \operatorname{lop}(S) \stackrel{S . u}{\rightarrow} \mathrm{w}$. If 


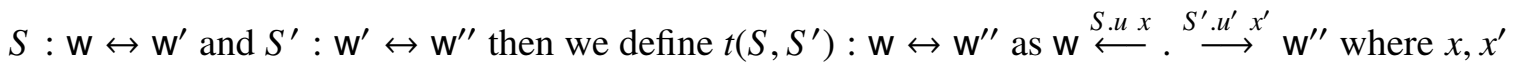
complete $S . u^{\prime}$ and $S^{\prime} . u$ to a pullback square.

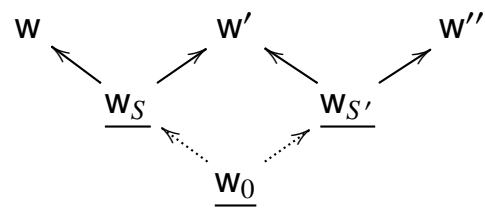

We assume a fixed choice of such completions to pullback squares. We do not assume that the $t$-operation is associative or satisfies any other laws.

Definition 9.1. A parametric square consists of two spans $S: \mathrm{w} \leftrightarrow \mathrm{w}^{\prime}$ and $S_{1}: \mathrm{w}_{1} \leftrightarrow \mathrm{w}_{1}^{\prime}$ and two morphisms $u: \mathrm{w} \rightarrow \mathrm{w}_{1}$ and $u^{\prime}: \mathrm{w}^{\prime} \rightarrow \mathrm{w}_{1}^{\prime}$ such that there exists a morphism $\underline{u}$ making the two squares in the following diagram pullbacks (thus in particular commute).

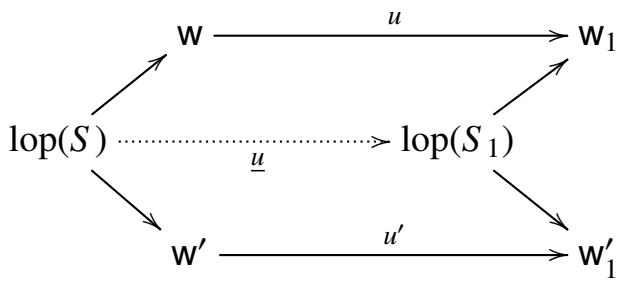

We use the notation $\left(u, u^{\prime}\right): S \rightarrow S_{1}$ in this situation.

Note that the witnessing morphism $\underline{u}$ is uniquely determined since we can complete $S_{1}$ to a pullback in which case $\underline{u}$ is the unique mediating morphism given by universal property of the latter pullback.

The reader is invited to check that under the interpretation of spans as partial bijections the presence of a parametric square $\left(u, u^{\prime}\right): S \rightarrow S^{\prime}$ asserts that $S^{\prime}$ is obtained from $S$ by consistent renaming followed by the addition of links and "garbage". In the following diagram the left diagram is parametric and the right one is not. In particular, the value 2 is mapped to 2 and 3 in the diagram to the right (as illustrated in red).
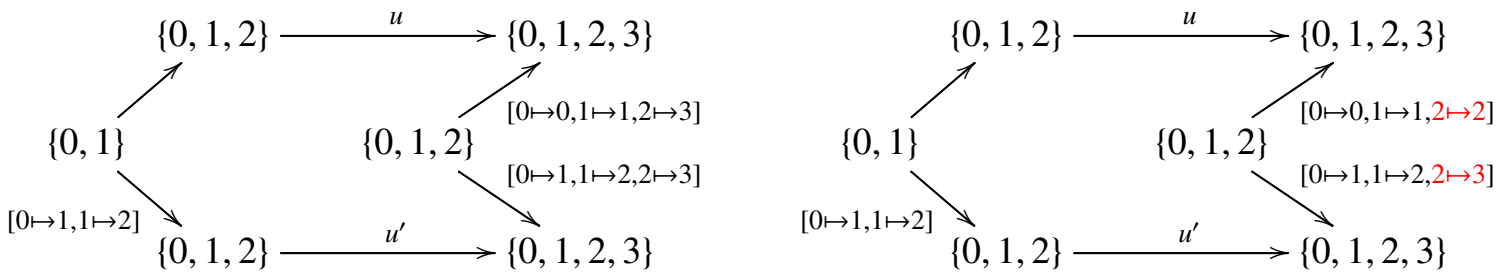

We also note that if $S, S^{\prime}: \mathrm{w} \leftrightarrow \mathrm{w}^{\prime}$ then (id,id) :S $\rightarrow S^{\prime}$ is a parametric square if and only if there exists an isomorphism $t: \operatorname{lop}(S) \rightarrow \operatorname{lop}\left(S^{\prime}\right)$ such that $S^{\prime} . u t=S . u$ and $S^{\prime} . u^{\prime} t=S . u^{\prime}$. In this case, we call $S$ and $S^{\prime}$ isomorphic spans and write $S \cong S^{\prime}$. Notice that for any span $S:$ w $\leftrightarrow$ w' $^{\prime}$ we have $t\left(S, r\left(\mathrm{w}^{\prime}\right)\right) \cong S \cong t\left(r(\mathrm{w}), \mathrm{w}^{\prime}\right)$ as well as other properties, such as associativity of $t(\cdot, \cdot)$ up to $\cong$.

Definition 9.2. A parametric functor is a set-valued functor on the category of worlds (a set $A \mathrm{w}$ for each world $\mathrm{w}$ and functorial transition functions $A u: A \mathrm{w} \rightarrow A \mathrm{w}^{\prime}$ when $\left.u: \mathrm{w} \rightarrow \mathrm{w}^{\prime}\right)$ together with a relation $A S \subseteq A \mathrm{w} \times A \mathrm{w}^{\prime}$ for each span $S: \mathrm{w} \leftrightarrow \mathrm{w}^{\prime}$. It is required that $\operatorname{Ar}(w)$ is the equality relation on $A$ w. 
9.2. Parametric Setoid-Valued Functors. Our aim is now to define a proof-relevant version of parametric functors: parametric SVFs. One way to go about this would be to identify a relational structure, i.e. an internal reflexive graph in the category of setoids, and then define parametric functors in the way that outlined by Stark [Sta94, Section 4.3]. Here, we prefer to take a different approach which gives a slightly richer structure. Namely, we consider relations that admit composition and taking opposites. This will lead to a logical relation that is both transitive and symmetric, and it appears that proof-relevance plays an important role in making that possible.

This construction could be given "decent categorical credentials" [Sta94, Section 4.3] by identifying an internal bicategory (as opposed to a reflexive graph) in the category of setoids, but we prefer to give a direct and elementary presentation which has the additional advantage that we can economise some data, namely the proof components of the relevant setoids, as those can be recovered from the relational structure by taking the identity extension property $(\operatorname{Ar}(\mathrm{w})=i d(A \mathrm{w}))$ as the very definition of (proof-relevant!) equality on the cpo Aw.

So, just like an SVF, a parametric SVF, $A$, specifies a predomain $A w$ for each w, and for each $u: \mathrm{w} \rightarrow \mathrm{w}^{\prime}$ a continuous function $A u: A \mathrm{w} \rightarrow A \mathrm{w}^{\prime}$. This time, however, we have a "heterogeneous equality" allowing one to compare elements of two different worlds without the need to transport them to a larger common world as done in SVFs. Thus, a parametric SVF has for each span $S: \mathrm{w} \leftrightarrow \mathbf{w}^{\prime}$ and elements $a \in A \mathrm{w}, a^{\prime} \in A \mathrm{w}^{\prime}$, a set of "proofs" $A S\left(a, a^{\prime}\right)$ asserting equality of these elements. As in the case of SVFs, the set of tuples $\left(S, a, a^{\prime}, p\right)$ with $p \in A S\left(a, a^{\prime}\right)$ must carry a predomain structure. We also require this semantic equality to be reflexive, symmetric and transitive in an heterogeneous sense, thus employing the $r, s, t$ operations on spans defined above. Furthermore, the transition functions should behave functorially, as for SVFs, but this time in the sense of the "heterogeneous equality". Every SVF gives rise to a parametric SVF by instantiating the heterogeneous equality to the larger world, but not all parametric SVFs are of this form (see Example 9.6).

Definition 9.3. A parametric $S V F, A$, consists of the following data.

(1) For each world $w$ a predomain $A w$.

(2) For each $u: \mathrm{w} \rightarrow \mathrm{w}^{\prime}$ a continuous function $A u: A \mathrm{w} \rightarrow A \mathrm{w}^{\prime}$. We use the notation $u . a=A u(a)$.

(3) For each span $S: \mathrm{w} \leftrightarrow \mathrm{w}^{\prime}$ and $a \in A \mathrm{w}$ and $a^{\prime} \in A \mathrm{w}^{\prime}$ a set $A S\left(a, a^{\prime}\right)$ such that the set of quadruples $\left(S, a, a^{\prime}, p\right)$ with $p \in A S\left(a, a^{\prime}\right)$ is a predomain with continuous second and third projections and discrete ordering in the first component.

(4) For each parametric square $\left(u, u^{\prime}\right): S \rightarrow S^{\prime}$ a continuous function

$$
A\left(u, u^{\prime}\right): \Pi a \in A \operatorname{dom}(S) . \Pi a^{\prime} \in A \operatorname{dom}^{\prime}(S) . A S\left(a, a^{\prime}\right) \rightarrow A S^{\prime}\left(u \cdot a, u^{\prime} \cdot a^{\prime}\right)
$$

(5) For each parametric square (id,id) $: S \rightarrow S^{\prime}$ a continuous function

$$
A\left(S, S^{\prime}\right): \Pi a \in A \operatorname{dom}(S) . \Pi a^{\prime} \in A \operatorname{dom}^{\prime}(S) . A S\left(a, a^{\prime}\right) \rightarrow A S^{\prime}\left(a, a^{\prime}\right)
$$

(6) Continuous functions of the following types, witnessing reflexivity, symmetry and transitivity in the "heterogeneous sense":

$$
\begin{aligned}
& \Pi \mathrm{w} . \Pi a \in A \mathrm{w} . A r(\mathrm{w})(a, a) \\
& \Pi S . \Pi a \in A \operatorname{dom}(S) . \Pi a^{\prime} \in A \operatorname{dom}(S) . A S\left(a, a^{\prime}\right) \rightarrow A s(S)\left(a^{\prime}, a\right) \\
& \Pi \mathrm{w} \mathrm{w}^{\prime} \mathrm{w}^{\prime \prime} . \Pi S: \mathrm{w} \leftrightarrow \mathrm{w}^{\prime} . \Pi S^{\prime}: \mathrm{w}^{\prime} \leftrightarrow \mathrm{w}^{\prime \prime} . \Pi a \in A \mathrm{w} . \Pi a^{\prime} \in A \mathrm{w}^{\prime} . \Pi a^{\prime \prime} \in A \mathrm{w}^{\prime \prime} . \\
& \quad A S\left(a, a^{\prime}\right) \times A S^{\prime}\left(a^{\prime}, a^{\prime \prime}\right) \rightarrow A t\left(S, S^{\prime}\right)\left(a, a^{\prime \prime}\right)
\end{aligned}
$$

(7) Continuous functions of the following types, witnessing the functorial laws:

$$
\begin{aligned}
& \Pi \mathrm{w} . \Pi a \in A \mathrm{w} . A r(\mathrm{w})(a, i d . a) \\
& \Pi \mathrm{w}_{1} \mathrm{w}_{2} . \Pi u: \mathrm{w} \rightarrow \mathrm{w}_{1} . \Pi v: \mathrm{w}_{1} \rightarrow \mathrm{w}_{2} \cdot \operatorname{Ar}\left(\mathrm{w}_{2}\right)(v \cdot u \cdot a,(v u) \cdot a)
\end{aligned}
$$


By way of motivating axiom (5), suppose that $S, S^{\prime}: \mathrm{w} \leftrightarrow \mathrm{w}^{\prime}$ are isomorphic spans between $\mathrm{w}$ and $\mathrm{w}^{\prime}$ in the sense that $(i d, i d): S \rightarrow S^{\prime}$ where $t$ is the isomorphism associated to $(i d, i d)$. If we have (an element of) $A S\left(a, a^{\prime}\right)$, then axiom (4) suffices to establish $A(i d, i d)\left(a, a^{\prime}\right) \in A S^{\prime}\left(\right.$ id.a, id. $\left.a^{\prime}\right)$. But what we really want is (an element of) $A S^{\prime}\left(a, a^{\prime}\right)$, which is just what axiom (5) provides in the shape of $A\left(S, S^{\prime}\right)\left(a, a^{\prime}\right)$. Without axiom (5), one can get quite close: using axioms (6) and (7) one can get (an element of) $\operatorname{At}\left(r(\mathrm{w}), t\left(S^{\prime}, s(r(\mathrm{w}))\right)\right)\left(a, a^{\prime}\right)$, for example. But without explicitly postulating axiom (5), as we do, or making extra assumptions on the $t(-,-)$ or $i d .-$ operations, it seems impossible to reach $A S^{\prime}\left(a, a^{\prime}\right)$. The following lemma is an instance of Axiom (5):

Lemma 9.4. If $S \cong S^{\prime}$ are isomorphic spans over $\mathrm{w}, \mathrm{w}^{\prime}$, there is a continuous function of type:

$$
\Pi a \in A \mathrm{w} . \Pi a^{\prime} \in A \mathrm{w}^{\prime} . A S\left(a, a^{\prime}\right) \rightarrow A S^{\prime}\left(a, a^{\prime}\right)
$$

Lemma 9.5. Let A be a parametric SVF. We have a continuous function of type:

$$
\Pi a \in A \mathrm{w} . \Pi \mathrm{w}_{1} . \Pi u: \mathrm{w} \rightarrow \mathrm{w}_{1} . A S(a, u . a)
$$

where $S$ is $\mathrm{w} \stackrel{\text { id }}{\leftarrow} \mathrm{w} \stackrel{u}{\rightarrow} \mathrm{w}_{1}$.

Proof. We use the parametric square $(i d, u): S_{0} \rightarrow S$ where $S_{0}$ is $\mathrm{w} \stackrel{i d}{\leftarrow} \mathrm{w} \stackrel{i d}{\rightarrow} \mathrm{w}$.

Every parametric SVF also is a plain SVF where we just define $A \mathrm{w}\left(a, a^{\prime}\right)=\operatorname{Ar}(\mathrm{w})\left(a, a^{\prime}\right)$ and quotient the transition maps $A u$ by pointwise $\sim$-equivalence.

But also every SVF $A$ can be extended to a parametric SVF: first fix a particular choice of transition functions $A u: A \mathrm{w} \rightarrow A \mathrm{w}^{\prime}$ when $u: \mathrm{w} \rightarrow \mathrm{w}^{\prime}$. Now define $A S\left(a, a^{\prime}\right)=A \overline{\mathrm{w}}\left(x \cdot a, x^{\prime} \cdot a^{\prime}\right)$ where $\overline{\mathrm{w}}$ is the apex of a completion of $S$ to a minimal pullback and $x: \operatorname{dom}(S) \rightarrow \overline{\mathrm{w}}, x^{\prime}: \operatorname{dom}^{\prime}(S) \rightarrow \overline{\mathrm{w}}$ are the corresponding maps.

However, this correspondence is not one-to-one. For a concrete counterexample, consider the following example which also lies at the heart of the justification of "Equivalence 12" with parametric functors.

Example 9.6. The parametric SVF $[N \Rightarrow B]$ is defined by $[N \Rightarrow B] \mathrm{w}=2^{\mathrm{w}}$ (functions from $\mathrm{w}$ to \{true, false\}) and

$$
[N \Rightarrow B] S\left(f, f^{\prime}\right)= \begin{cases}\{\star\} & \text { if } \forall n \in \operatorname{lop}(S) . f(S . u(n))=f^{\prime}\left(S . u^{\prime}(n)\right) \\ \emptyset & \text { otherwise }\end{cases}
$$

Now, let $S$ be $\{0\} \leftarrow \emptyset \rightarrow \emptyset$ and put $f(x)=$ “ $x=0$ " and $f^{\prime}(x)=$ false. We have $[N \Rightarrow B] S\left(f, f^{\prime}\right)$, i.e., $[N \Rightarrow B] S\left(f, f^{\prime}\right)=\{\star\}$, thus $f$ and $f^{\prime}$ are considered equal above span $S$. On the other hand, if we complete $S$ to a minimal pullback by $\{0\} \stackrel{1}{\rightarrow}\{0\} \stackrel{x^{\prime}}{\leftarrow} \emptyset$ then $[N \Rightarrow B] r(\{0\})\left(f, x^{\prime} . f\right)=\emptyset$, i.e., $f$ and $f^{\prime}$ are not equal when regarded over the least common world, namely $\{0\}$.

Definition 9.7. A parametric natural transformation, $f$, from parametric SVF $A$ to $B$ consists of two continuous functions

$$
\begin{aligned}
& f_{0}: \Pi w . A \mathrm{w} \rightarrow B \mathrm{w} \\
& f_{1}: \Pi S . \Pi a \in \operatorname{dom}(S) . \Pi a^{\prime}: \operatorname{dom}^{\prime}(S) . A S\left(a, a^{\prime}\right) \rightarrow B S\left(f_{0} \operatorname{dom}(S)(a), f_{0} \operatorname{dom}^{\prime}(S)\left(a^{\prime}\right)\right)
\end{aligned}
$$

As usual we refer both $f_{0}$ and $f_{1}$ as $f$. Two parametric natural transformations $f, f^{\prime}: A \rightarrow B$ are identified if there is a continuous function of type

$$
\Pi \mathrm{w} . \Pi a \in A \mathrm{w} . \operatorname{Br}(\mathrm{w})\left(f \mathrm{w}(a), f^{\prime} \mathrm{w}(a)\right)
$$

The identification of "pointwise equal" parametric natural transformations is meaningful as follows: 
Lemma 9.8. Let $f$ and $f^{\prime}$ be representatives of the same parametric natural transformation $A \rightarrow B$. There then is a continuous function of the following type:

$$
\Pi S . \Pi a \in \operatorname{dom}(S) . \Pi a^{\prime} \in \operatorname{dom}^{\prime}(S) . A S\left(a, a^{\prime}\right) \rightarrow B S\left(f \operatorname{dom}(S)(a), f^{\prime} \operatorname{dom}^{\prime}(S)\left(a^{\prime}\right)\right)
$$

Proof. Given $S, a, a^{\prime}$, and $p \in A S\left(a, a^{\prime}\right)$ we obtain $B S\left(f \operatorname{dom}(S)(a), f \operatorname{dom}^{\prime}(S)\left(a^{\prime}\right)\right)$ and we also obtain $\operatorname{Br}\left(\operatorname{dom}^{\prime}(S)\right)\left(f \operatorname{dom}^{\prime}(S)\left(a^{\prime}\right), f \operatorname{dom}^{\prime}(S)\left(a^{\prime}\right)\right)$ since $f$ and $f^{\prime}$ are pointwise equal. We conclude by transitivity and Lemma 9.4 .

Lemma 9.9. If $f: A \rightarrow B$ is a parametric natural transformation then there are continuous functions of the following types

$$
\begin{aligned}
& \prod \mathrm{w} \mathrm{w}_{1} . \Pi u: \mathrm{w} \rightarrow \mathrm{w}_{1} . \Pi a \in A \mathrm{w} \cdot \operatorname{Br}\left(\mathrm{w}_{1}\right)\left(u \cdot f \mathrm{w}(a), f \mathrm{w}_{1}(u \cdot a)\right) \\
& \prod \mathrm{w} . \Pi a a^{\prime} \in A \mathrm{w} \cdot \operatorname{Ar}(\mathrm{w})\left(a, a^{\prime}\right) \rightarrow \operatorname{Br}(\mathrm{w})\left(f \mathrm{w}(a), f \mathrm{w}\left(a^{\prime}\right)\right)
\end{aligned}
$$

Proof. Fix $u, a$ and w. Lemma 9.5 furnishes an element of $A S(a, u . a)$ where $S$ is $\mathrm{w} \stackrel{1}{\leftarrow} \mathrm{w} \stackrel{u}{\rightarrow} \mathrm{w}_{1}$. Since $f$ is a parametric natural transformation, we then get an element of $B S\left(f \mathrm{w}(a), f \mathrm{w}_{1}(u \cdot a)\right)$. We then get the desired element of $B r\left(\mathrm{w}_{1}\right)\left(u . f \mathrm{w}(a), f \mathrm{w}_{1}(u . a)\right)$ by applying parametricity of $B$ to the parametric square $(u, 1): S \rightarrow r\left(\mathrm{w}_{1}\right)$.

Theorem 9.10. The parametric SVFs with parametric natural transformations form a cartesian closed category with fixpoint operator obeying the laws from Theorems $2.3 \mathcal{E} 5.3$. There is a strong monad $T$ on this category where

$T A \mathrm{w}=\left\{\left(\mathrm{w}_{1}, a\right) \mid \mathrm{w} \subseteq \mathrm{w}_{1} \wedge a \in A \mathrm{w}_{1}\right\}_{\perp}$

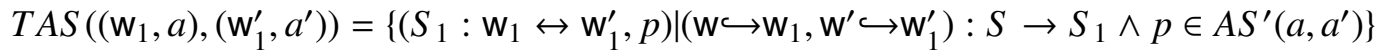

Proof (sketch). The interesting bit is the proof of transitivity for the monad, which seems to rely in an essential manner on proof relevance. Suppose that $S: \mathrm{w} \leftrightarrow \mathrm{w}^{\prime}$ and $S^{\prime}: \mathrm{w}^{\prime} \leftrightarrow \mathrm{w}^{\prime \prime}$ and that $\left(\mathrm{w}_{1}, a\right) \in T A \mathrm{w}$ and $\left(\mathrm{w}_{1}^{\prime}, a^{\prime}\right) \in T A \mathrm{w}^{\prime}$ and $\left(\mathrm{w}_{1}^{\prime \prime}, a^{\prime \prime}\right) \in T A \mathrm{w}^{\prime \prime}$. Furthermore, suppose that $\left(S_{1}, p\right) \in$ $T A S\left(\left(\mathrm{w}_{1}, a\right),\left(\mathrm{w}_{1}^{\prime}, a^{\prime}\right)\right)$ and $\left(S_{1}^{\prime}, p^{\prime}\right) \in T A S^{\prime}\left(\left(\mathrm{w}_{1}^{\prime}, a^{\prime}\right),\left(\mathrm{w}_{1}^{\prime \prime}, a^{\prime \prime}\right)\right)$.

Now, by definition, we have $S_{1}: \mathrm{w}_{1} \leftrightarrow \mathrm{w}_{1}^{\prime}$ and $S_{1}^{\prime}: \mathrm{w}_{1}^{\prime} \leftrightarrow \mathrm{w}_{1}^{\prime \prime}$ and also $p \in A S_{1}\left(a, a^{\prime}\right)$ and $p^{\prime} \in A S_{1}^{\prime}\left(a^{\prime}, a^{\prime \prime}\right)$. We thus obtain (an element of) $\operatorname{At}\left(S_{1}, S_{1}^{\prime}\right)\left(a, a^{\prime \prime}\right)$ and this, together with $t\left(S_{1}, S_{1}^{\prime}\right)$ furnishes the required proof.

Remark 9.11. Notice that if the extensions $w_{1}$ were existentially quantified, as in more traditional non-proof-relevant formulations of Kripke logical relations (e.g. that of Stark [Sta94, Section 4.1]), then the transitivity construction in the above proof would not have been possible because we would have no guarantee that the existential witnesses used in the two assumptions are the same.

9.3. Private Name Equation. We now return to our motivating equivalence, illustrating that a function value may encapsulate a freshly generated name without revealing it to the context:

$$
\text { (let } n \Leftarrow \text { new in } \lambda x \text {. }(x=n))=(\lambda x \text {.false }) \text {. }
$$


Writing $c$ and $c^{\prime}$ for the LHS and RHS of the above, the equivalence proof is based on the following diagram:

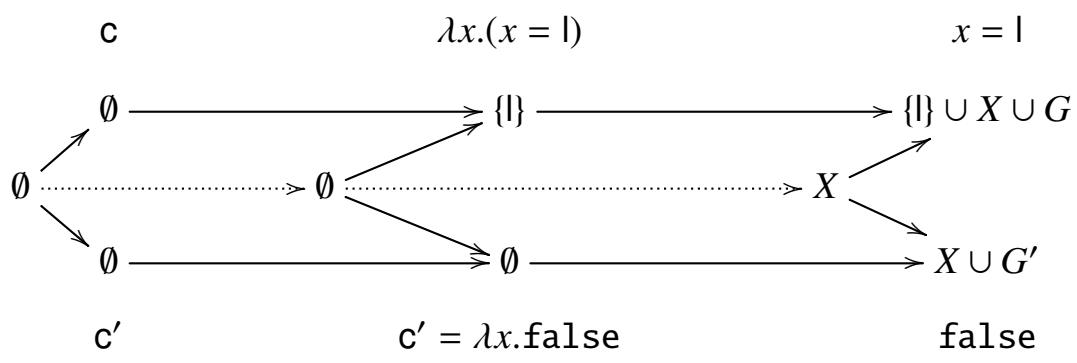

We show that $c$ and $c^{\prime}$ are equivalent in the trivial span to the left. For the generation of the fresh value in $\mathrm{c}$, we choose the extension of the worlds with the fresh value I, the second span shown in the diagram. Now it remains to prove that $\lambda x . x=I$ and $c^{\prime}$ are equivalent above the latter. This means that for any extension of worlds, $x=I$ and false should be related. Consider the extension of worlds in the right-most span in the diagram above. The names in $X$ denote the common names, while $G$ and $G^{\prime}$ the spurious names created. Notice that I is not in the low point of the third span because the squares with vertices $\emptyset, X,\{\mid\} \cup X \cup G,\{\mid\}$ and $\emptyset, X, X \cup G^{\prime}, \emptyset$ are pullbacks as by Definition 9.1. Thus, the value of $x$ cannot be $I$ and $x=I$ is indeed equal to false.

\section{Discussion}

We have introduced proof-relevant logical relations and shown how they may be used to model and reason about simple equivalences in a higher-order language with recursion and name generation. A key innovation compared with previous functor category models is the use of functors valued in setoids (which are here also built on predomains), rather than plain sets. One payoff is that we can work with a direct style model rather than one based on continuations (which, in the absence of control operators in the language, is less abstract).

The technical machinery used here is not entirely trivial, and the reader might be forgiven for thinking it slightly excessive for such a simple language and rudimentary equations. However, our aim has not been to present impressive new equivalences, but rather to present an accessible account of how the idea of proof relevant logical relations works in a simple setting. The companion paper [BHN14] gives significantly more advanced examples of applying the construction to reason about equivalences justified by abstract semantic notions of effects and separation, but the way in which setoids are used is there potentially obscured by the details of, for example, much more sophisticated categories of worlds. Our hope is that this account will bring the idea to a wider audience, make the more advanced applications more accessible, and inspire others to investigate the construction in their own work.

Thanks to Andrew Kennedy for numerous discussions, to the referee who first suggested that we write up the details of how proof-relevance applies to pure name generation, and to the referees of the present paper for their many helpful suggestions.

\section{REFERENCES}

$\left[\mathrm{AGM}^{+}\right.$04] S. Abramsky, D. R. Ghica, A. S. Murawski, C.-H. L. Ong, and I. D. B. Stark. Nominal games and full abstraction for the nu-calculus. In Proc. 19th Annual IEEE Symposium on Logic in Computer Science (LICS '04). IEEE Computer Society, 2004. 
[BB06] N. Bohr and L. Birkedal. Relational reasoning for recursive types and references. In Proc. Fourth Asian Symposium on Programming Languages and Systems (APLAS '06), volume 4279 of LNCS. Springer, 2006.

[BCP03] Gilles Barthe, Venanzio Capretta, and Olivier Pons. Setoids in type theory. J. Funct. Program., 13(2):261-293, 2003.

[BCRS98] Lars Birkedal, Aurelio Carboni, Giuseppe Rosolini, and Dana S. Scott. Type theory via exact categories. In Proc. 13th Annual IEEE Symposium on Logic in Computer Science (LICS '98). IEEE Computer Society, 1998.

[BÉ93] Stephen L. Bloom and Zoltán Ésik. Iteration Theories - The Equational Logic of Iterative Processes. EATCS Monographs on Theoretical Computer Science. Springer, 1993.

[BHN14] Nick Benton, Martin Hofmann, and Vivek Nigam. Abstract effects and proof-relevant logical relations. In Proc. 41st ACM SIGPLAN-SIGACT Symposium on Principles of Programming Languages (POPL '14). ACM, 2014.

[BK13] N. Benton and V. Koutavas. A mechanized bisimulation for the nu-calculus. Higher-Order and Symbolic Computation, 2013. To appear.

[BKBH07] Nick Benton, Andrew Kennedy, Lennart Beringer, and Martin Hofmann. Relational semantics for effect-based program transformations with dynamic allocation. In Proc. Ninth International ACM SIGPLAN Symposium on Principles and Practice of Declarative Programming (PPDP '07). ACM, 2007.

[BKHB06] Nick Benton, Andrew Kennedy, Martin Hofmann, and Lennart Beringer. Reading, writing and relations: Towards extensional semantics for effect analyses. In Proc. Fourth Asian Symposium on Programming Languages and Systems (APLAS '06), volume 4279 of LNCS. Springer, 2006.

[BL05] Nick Benton and Benjamin Leperchey. Relational reasoning in a nominal semantics for storage. In Proc. Seventh International Conference on Typed Lambda Calculi and Applications (TLCA '05), volume 3461 of LNCS. Springer, 2005.

[CFS87] Aurelio Carboni, Peter J. Freyd, and Andre Scedrov. A categorical approach to realizability and polymorphic types. In Proc. Third Workshop on Mathematical Foundations of Programming Language Semantics (MFPS '87), volume 298 of LNCS. Springer, 1987.

[GP02] Murdoch Gabbay and Andrew M. Pitts. A new approach to abstract syntax with variable binding. Formal Asp. Comput., 13(3-5):341-363, 2002.

[nLa] nLab. cartesian closed category. https://ncatlab.org/nlab/show/cartesian+closed+category\# exponentials_of_cartesian_closed_categories.

[PS93] Andrew M. Pitts and Ian D. B. Stark. Observable properties of higher-order functions that dynamically create local names, or what's new? In Proc. 18th International Symposium on Mathematical Foundations of Computer Science (MFCS '93), volume 711 of LNCS. Springer, 1993.

[PS98] Andrew Pitts and Ian Stark. Operational reasoning for functions with local state. In Higher Order Operational Techniques in Semantics, pages 227-273. Cambridge University Press, 1998.

[Shi04] Mark R. Shinwell. The Fresh Approach: functional programming with names and binders. PhD thesis, University of Cambridge, 2004.

[SP00] Alex K. Simpson and Gordon D. Plotkin. Complete axioms for categorical fixed-point operators. In LICS, pages 30-41. IEEE Computer Society, 2000.

[SP05] Mark R. Shinwell and Andrew M. Pitts. On a monadic semantics for freshness. Theor. Comput. Sci., 342(1):2855, 2005.

[SPG03] Mark R. Shinwell, Andrew M. Pitts, and Murdoch J. Gabbay. FreshML: Programming with binders made simple. In Proc. Eighth ACM SIGPLAN International Conference on Functional programming (ICFP '03). ACM, 2003.

[Sta94] I. D. B. Stark. Names and Higher-Order Functions. PhD thesis, University of Cambridge, Cambridge, UK, December 1994. Also published as Technical Report 363, University of Cambridge Computer Laboratory.

[Tze12] N. Tzevelekos. Program equivalence in a simple language with state. Computer Languages, Systems and Structures, 38(2), 2012. 